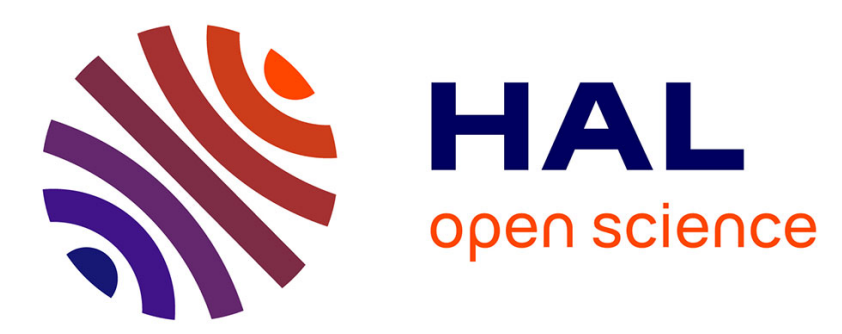

\title{
Quantum interference effect for two atoms excited by molecular photodissociation: a theoretical analysis
}

\author{
Philippe Grangier, Jacques Vigué
}

\section{To cite this version:}

Philippe Grangier, Jacques Vigué. Quantum interference effect for two atoms excited by molecular photodissociation: a theoretical analysis. Journal de Physique, 1987, 48 (5), pp.781-796. 10.1051/jphys:01987004805078100 . jpa-00210498

\section{HAL Id: jpa-00210498 https://hal.science/jpa-00210498}

Submitted on 1 Jan 1987

HAL is a multi-disciplinary open access archive for the deposit and dissemination of scientific research documents, whether they are published or not. The documents may come from teaching and research institutions in France or abroad, or from public or private research centers.
L'archive ouverte pluridisciplinaire HAL, est destinée au dépôt et à la diffusion de documents scientifiques de niveau recherche, publiés ou non, émanant des établissements d'enseignement et de recherche français ou étrangers, des laboratoires publics ou privés. 
Classification

Physics Abstracts

33.50D $-03.65 \mathrm{~B}-33.80 \mathrm{G}-35.80$

\title{
Quantum interference effect for two atoms excited by molecular photodissociation : a theoretical analysis
}

\author{
P. Grangier and J. Vigué $\left(^{+}\right)$ \\ Institut d'Optique (*), B.P. 43, Centre Universitaire d'Orsay, 91406 Orsay Cedex, France \\ $\left({ }^{+}\right)$Laboratoire de Spectroscopie Hertzienne $(*)$, Ecole Normale Supérieure, 24 rue Lhomond, \\ 75231 Paris Cedex 05, France
}

(Reçu le 2 octobre 1986, accepté le 5 janvier 1987)

\begin{abstract}
Résumé. - Nous présentons l'analyse théorique d'une expérience, dans laquelle nous avons observé une modulation temporelle dans la lumière de fluorescence d'un système de deux atomes, obtenus par photodissociation de molécules $\mathrm{Ca}_{2}$ par une impulsion laser. Le calcul est d'abord effectué sur un modèle simple, dans lequel on considère deux atomes couplés au champ électromagnétique quantifié. Nous discutons ensuite la pertinence de ce modèle, du point de vue de la physique moléculaire. Enfin, nous montrons que l'on obtient un bon accord entre les résultats théoriques et expérimentaux, à condition de prendre en compte les niveaux quasi liés de l'état fondamental de la molécule photodissociée.
\end{abstract}

\begin{abstract}
We present a theoretical analysis of a previous experiment, in which a modulation in the timeresolved atomic fluorescence light following the photodissociation of $\mathrm{Ca}_{2}$ molecules by a pulsed laser was observed. First a calculation is performed on a simple model, involving two atoms coupled with the quantized radiation field. Then the validity of this model is discussed on molecular physics grounds. Finally, a quantitative analysis of the experiment is presented ; a good agreement is obtained if quasi-bound levels of the molecular ground state are taken into account.
\end{abstract}

\section{Introduction.}

In a recent paper [1], we presented experimental evidence for a quantum interference effect occurring in the photodissociation of a diatomic homonuclear molecule $\mathbf{A}_{2}$. This photodissociation yields two atoms recoiling in opposite directions, one in an excited state $\left(A^{*}\right)$, and one in the ground state $(A)$. Either of the two atoms can actually be excited, and subsequently reemit a photon at the atomic frequency $\omega_{0}$, so one must consider two interfering paths for the whole process :

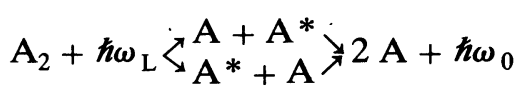

( $\omega_{\mathrm{L}}$ refers to the photodissociating laser light).

The interference effect appears as a modulation of the probability $P(\tau)$ of detecting the fluorescence photon $\hbar \omega_{0}$ at the delay $\tau$ after the dissociation effected by a laser pulse at $\tau=0$ ( $\tau$ is a retarded time with propagation delay subtracted). The basic

(*) Laboratoires associés au C.N.R.S. JOURNAL DE PHYSIQUE. - T. 48, N 5, MAI 1987 experimental setup (Fig. 1) involves a molecular beam, at right angle to the dissociating laser beam. The fluorescent light is collected at right angle to both the atomic beam and the electric field $\mathbf{E}_{\mathrm{L}}$ of the

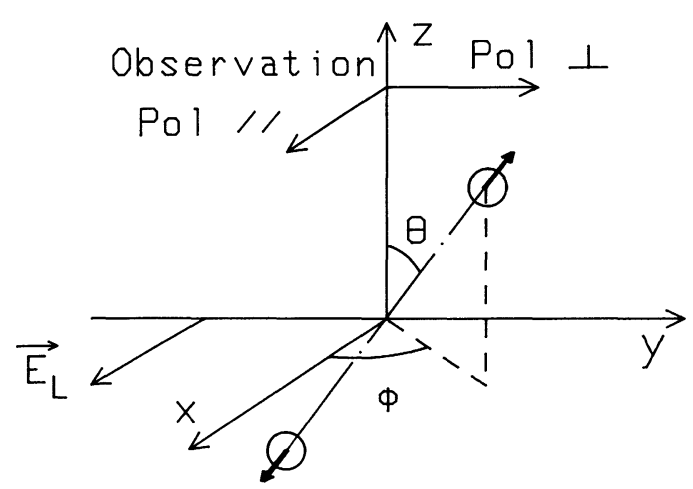

Fig. 1. - Schematic experimental set-up. The atomic and laser beams are respectively along $O x$ and $O y$; the fluorescence light is collected along $\mathrm{O} z$, through a polarizer that can be set parallel (//) or orthogonal $(\perp)$ to the electric field $E_{\mathrm{L}}$ of the photodissociating laser. The angles $\theta$ and $\phi$ characterize the recoil direction of the atoms. 
laser, through a polarizer that can be set parallel (//) or orthogonal $(\perp)$ to $\mathbf{E}_{\mathrm{L}}$ (see Fig. 1).

In this paper, we present a quantum mechanical calculation of the observed signal, quantizing the electromagnetic field and the motion of both atoms. In the first part, of the paper, this calculation is performed on a somehow idealized model. In the second part, we discuss the validity of this model on molecular physics grounds, and in the third part some peculiar points relevant to experiments are considered. Our theoretical approach uses the Heisenberg equations for the field and atomic operators, which allows to exhibit the relationship between the classical and quantum treatments of the interference effect. On the other hand, we will analyse also some consequences of the fact that a single photon is involved in the process described by equation (1) ; this step requires obviously a quantum treatment of the field.

\section{Quantum mechanical model.}

1.1 Notations. - We consider two identical atoms $a$ and $b$, interacting with the quantized electromagnetic field. The Hamiltonian of this system is the sum of four parts :

$$
H=H_{\mathrm{a}}+H_{\mathrm{b}}+H_{\mathrm{R}}+V
$$

where $H_{\mathrm{a}}$ and $H_{\mathrm{b}}$ are atomic Hamiltonians, including kinetic energy terms, $H_{\mathrm{R}}$ is the radiation Hamiltonian and $V$ is the atoms-field coupling.

The atoms $\mathrm{a}$ and $\mathrm{b}$ are considered as two-levels systems, (excited state : $|\mathrm{e}\rangle$, ground state : $|\mathrm{g}\rangle$ ), and the operators $\mathbf{R}_{\mathrm{a}}$ and $\mathbf{R}_{\mathrm{b}}$ (resp. $\mathbf{P}_{\mathrm{a}}$ and $\mathbf{P}_{\mathrm{b}}$ ) are attributed to the positions (resp. momenta) of the centres of mass of both atoms. In this model, only radiative interactions between the atoms will be considered ; in particular, the internuclear distance $\delta$ will be assumed large enough to ignore chemical bindings.

We have thus :

$$
\begin{aligned}
& H_{\mathrm{a}}=\frac{\mathbf{P}_{\mathrm{a}}^{2}}{2 M}+\hbar \omega_{0} S_{z_{\mathrm{a}}} \\
& H_{\mathrm{b}}=\frac{\mathbf{P}_{\mathrm{b}}^{2}}{2 M}+\hbar \omega_{0} S_{z_{\mathrm{b}}}
\end{aligned}
$$

where $\omega_{0}$ is the atomic resonance frequency and $S_{z_{i}}$ the $z$-component of the effective-spin operator for each atom $(i=\mathbf{a}$ or $\mathrm{b})$ :

$$
\begin{gathered}
S_{z_{i}}=\frac{1}{2}\left(S_{i}^{+} S_{i}^{-}-S_{i}^{-} S_{i}^{+}\right) \\
S_{i}^{+}=\left|\mathrm{e}_{i}\right\rangle\left\langle\mathrm{g}_{i}\left|\quad S_{i}^{-}=\right| \mathrm{g}_{i}\right\rangle\left\langle\mathrm{e}_{i}\right| .
\end{gathered}
$$

The electromagnetic radiation field is quantized on a complete set of orthogonal field distribution $\boldsymbol{\xi}_{\lambda}(\mathbf{R})$, for example plane waves of wave vector $\mathbf{k}_{\lambda}$, angular frequency $\omega_{\lambda}=c\left|\mathbf{k}_{\lambda}\right|$, and polarization $\varepsilon_{\lambda}$. The Hamiltonian of the free radiation field is thus :

$$
H_{\mathrm{R}}=\sum_{\lambda} \hbar \omega_{\lambda} a_{\lambda}^{+} a_{\lambda}
$$

where $a_{\lambda}$ and $a_{\lambda}^{+}$are respectively the destruction and creation operators of one photon in the mode $\lambda$.

The positive and negative frequency components of the electric field are given by :

$$
\left\{\begin{array}{l}
\mathbf{E}^{+}(\mathbf{R})=\sum_{\lambda} \boldsymbol{\xi}_{\lambda}(\mathbf{R}) a_{\lambda} \\
\mathbf{E}^{-}(\mathbf{R})=\sum_{\lambda} \boldsymbol{\xi}_{\lambda}^{*}(\mathbf{R}) a_{\lambda}^{+}
\end{array}\right.
$$

where

$$
\xi_{\lambda}(\mathbf{R})=i \sqrt{\frac{\hbar \omega_{\lambda}}{2 \varepsilon_{0} V}} \varepsilon_{\lambda} \mathrm{e}^{i \mathbf{k}_{\lambda} \cdot \mathbf{R}} .
$$

The atoms-field coupling at the electric dipole approximation can then be shown to be [2] :

$$
V=-\mathbf{D}_{\mathrm{a}} \cdot \mathbf{E}\left(\mathbf{R}_{\mathrm{a}}\right)-\mathbf{D}_{\mathrm{b}} \cdot \mathbf{E}\left(\mathbf{R}_{\mathrm{b}}\right)
$$

where $\mathbf{D}_{\mathrm{a}}$ (resp. $\mathbf{D}_{\mathrm{b}}$ ) is the dipole operator of atom a (resp. atom b).

More precisely, we have for atom $i$ :

$$
\mathbf{D}_{i}=\mathbf{d}_{\mathrm{eg}}\left(S_{i}^{+}+S_{i}^{-}\right)
$$

where $\mathbf{d}_{\mathrm{eg}}=\langle\mathrm{e}|\mathbf{D}| \mathrm{g}\rangle$ is assumed to be a real vector depending on the levels $|\mathrm{e}\rangle$ and $|\mathrm{g}\rangle$. We define $g_{\lambda}$ as the quantity :

$$
\hbar g_{\lambda}=-\sqrt{\frac{\hbar \omega_{\lambda}}{2 \varepsilon_{0} V}} \varepsilon_{\lambda} \cdot \mathbf{d}_{\mathrm{eg}}
$$

and we get, using the rotating wave approximation, the normal-ordered coupling :

$$
\begin{aligned}
& V=i \sum_{\lambda} \hbar g_{\lambda} {\left[\left\{\mathrm{e}^{i \mathbf{k}_{\lambda} \cdot \mathbf{R}_{\mathrm{a}}} S_{\mathrm{a}}^{+}+\mathrm{e}^{i \mathbf{k}_{\lambda} \cdot \mathbf{R}_{\mathrm{b}}} S_{\mathrm{b}}^{+}\right\} a_{\lambda}-\right.} \\
&\left.-a_{\lambda}^{+}\left\{\mathrm{e}^{-i \mathbf{k}_{\lambda} \cdot \mathbf{R}_{\mathrm{a}}} S_{\mathrm{a}}^{-}+\mathrm{e}^{-i \mathbf{k}_{\lambda} \cdot \mathbf{R}_{\mathrm{b}}} S_{\mathrm{b}}^{-}\right\}\right] .
\end{aligned}
$$

We will suppose that just after the end of the laser pulse, the initial state vector $\left|\psi_{\text {in }}\right\rangle$ describing the two atoms in vacuum radiation field can be factorized in three parts (see discussion in Part 2) :

$$
\left|\psi_{\text {in }}\right\rangle=\left|\psi_{\text {int }}\right\rangle\left|\psi_{\text {ext }}\right\rangle\left|V_{\text {ac }}\right\rangle \text {. }
$$

- The first part corresponding to the internal degrees of freedom of the atoms is given by :

$$
\left|\psi_{\text {int }}\right\rangle=\frac{1}{\sqrt{2}}\left\{\left|g_{\mathrm{a}} e_{\mathrm{b}}\right\rangle+\varepsilon\left|e_{\mathrm{a}} g_{\mathrm{b}}\right\rangle\right\}
$$

where $\varepsilon$ is equal to +1 or -1 according to the molecular parity ( $u$ or $g$ ) of the dissociating state. 
- The second part corresponds to the external degrees of freedom. Actually, $\left|\psi_{\text {ext }}\right\rangle$ is a function of the ground molecular state involved in the photodissociation, and, due to the thermal populations of these states, the external degrees of freedom would be more adequately described by a density matrix. In this part of the paper, we shall consider the contribution of a single ground state level, i.e. we assume that the description by $\left|\psi_{\text {ext }}\right\rangle$ is valid. However, the average over the molecular ground state population will be carried out in part 2 .

Without specifying explicitly $\left|\psi_{\text {ext }}\right\rangle$, we assume that the dispersion of the relative position $\left(\mathbf{R}_{a}-\mathbf{R}_{b}\right)$ of the atoms is very small compared to the wavelength $\lambda_{0}$ of the emitted radiation. This hypothesis will be qualitatively justified in part 2 .

- The third part is the vacuum state of the radiation field.

1.2 Heisenberg equations. - To proceed further, we adopt the Heisenberg representation, which is very convenient for displaying the relationship between the classical and quantum descriptions of the electromagnetic field. The state vector is thus kept constant, and equal to $\left|\psi_{\text {in }}\right\rangle$, while the operators $S^{+}, S^{-}, \mathbf{P}, \mathbf{R}, \mathrm{a}^{+}$, a are time dependent. The time evolution of these operators are $(i=\mathrm{a}$ or $\mathrm{b})$ :

$$
\begin{aligned}
& \frac{\mathrm{d}}{\mathrm{d} t} \mathbf{R}_{i}=\frac{\mathbf{P}_{i}}{\mathbf{M}} \\
& \frac{\mathrm{d}}{\mathrm{d} t} \mathbf{P}_{i}=\sum_{\lambda} g_{\lambda} \hbar \mathbf{k}_{\lambda} \times \\
& \times\left(S_{i}^{+} a_{\lambda} \mathrm{e}^{i \mathbf{k}_{\lambda} \cdot \mathbf{R}_{i}}+a_{\lambda}^{+} S_{i}^{-} \mathrm{e}^{-i \mathbf{k}_{\lambda} \cdot \mathbf{R}_{i}}\right) \\
& \frac{\mathrm{d}}{\mathrm{d} t} a_{\lambda}=i \omega_{\lambda} a_{\lambda}-g_{\lambda}\left(S_{\mathrm{a}}^{-} \mathrm{e}^{-i \mathbf{k}_{\lambda} \cdot \mathbf{R}_{\mathrm{a}}}+S_{\mathrm{b}}^{-} \mathrm{e}^{-i \mathbf{k}_{\lambda} \cdot \mathbf{R}_{\mathrm{b}}}\right) \\
& \frac{\mathrm{d}}{\mathrm{d} t} S_{i}^{-}=-i \omega_{0} S_{i}^{-}- \\
& -\sum_{\lambda} g_{\lambda}\left(2 S_{i}^{+} S_{i}^{-}-1\right) a_{\lambda} \mathrm{e}^{i \mathbf{k}_{\lambda} \cdot \mathbf{R}_{i}} .
\end{aligned}
$$

These equations can be recasted in a simpler form by separating the rapidly varying part of the operators (due to the free terms in the Hamiltonian) from the slowly varying terms (due to the coupling); we thus write :

$$
\begin{aligned}
S_{i}^{-} & =\mathrm{e}^{-i \omega_{0} t} \tilde{S}_{i}^{-} \\
a_{\lambda} & =\mathrm{e}^{-i \omega_{\lambda} t} \tilde{a}_{\lambda}
\end{aligned}
$$

and we get :

$\frac{\mathrm{d}}{\mathrm{d} t} \tilde{a}_{\lambda}=-g_{\lambda} \mathrm{e}^{i\left(\omega_{\lambda}-\omega_{0}\right) t}\left(\tilde{S}_{\mathrm{a}}^{-} \mathrm{e}^{-i \mathbf{k}_{\lambda} \mathbf{R}_{\mathrm{a}}}+\tilde{S}_{\mathrm{b}}^{-} \mathrm{e}^{-i \mathbf{k}_{\lambda} \cdot \mathbf{R}_{\mathrm{b}}}\right)$

$\frac{\mathrm{d}}{\mathrm{d} t} \tilde{S}_{i}^{-}=-\sum_{\lambda} g_{\lambda}\left(2 \tilde{S}_{i}^{+} \tilde{S}_{i}^{-}-1\right) a_{\lambda} \mathrm{e}^{i\left(\mathbf{k}_{\lambda} \cdot \mathbf{R}_{i}+\left(\omega_{0}-\omega_{\lambda}\right) t\right)}$.
1.3 Time EVolution of ATOMIC AND Field OPERATORS. - Integration of the first equation of system (13) yields :

$$
\begin{array}{r}
\tilde{a}_{\lambda}(t)=\tilde{a}_{\lambda}(0)-g_{\lambda} \int_{0}^{t}\left(\tilde{S}_{\mathrm{a}}^{-}\left(t^{\prime}\right) \mathrm{e}^{-i \mathbf{k}_{\lambda} \cdot \mathbf{R}_{\mathrm{a}}\left(t^{\prime}\right)}+\right. \\
\left.+\tilde{S}_{\mathrm{b}}^{-} \mathrm{e}^{-i \mathbf{K}_{\lambda} \mathbf{R}_{\mathrm{b}}\left(t^{\prime}\right)}\right) \times \mathrm{e}^{i\left(\omega_{\lambda}-\omega_{0}\right) t^{\prime}} \mathrm{d} t^{\prime} .
\end{array}
$$

Provided that this operator will be applied on the initial state $\left|\psi_{\text {in }}\right\rangle$, we can use the equality $a_{\lambda}(0)\left|\psi_{\text {in }}\right\rangle=0$ (the field is in the vacuum state at $t=0)$; the second equation of system (13) then yields $((i, j)=(\mathrm{a}, \mathrm{b})$ or $(\mathrm{b}, \mathrm{a}))$ :

$$
\begin{aligned}
& \frac{\mathrm{d}}{\mathrm{d} t} \tilde{S}_{i}^{-}(t)\left|\psi_{\text {in }}\right\rangle=\sum_{\lambda} g_{\lambda}^{2}\left(2 \tilde{S}_{i}^{+} \tilde{S}_{i}^{-}-1\right) \mathrm{e}^{i \mathbf{k}_{\lambda} \cdot \mathbf{R}_{i}(t)} \\
& \quad \times \int_{0}^{t}\left(\tilde{S}_{i}^{-}\left(t^{\prime}\right) \mathrm{e}^{-i \mathbf{k}_{\lambda} \cdot \mathbf{R}_{i}\left(t^{\prime}\right)}+\tilde{S}_{j}^{-}\left(t^{\prime}\right) \mathrm{e}^{-i \mathbf{k}_{\lambda} \cdot \mathbf{R}_{j}\left(t^{\prime}\right)}\right) \\
& \quad \times \mathrm{e}^{i\left(\omega_{\lambda}-\omega_{0}\right)\left(t^{\prime}-t\right)} \mathrm{d} t^{\prime}\left|\psi_{\text {in }}\right\rangle
\end{aligned}
$$

Equations (15) are the main equations describing the desexcitation of the two-atoms system. Before solving this system, let us notice that in the singleatom case (i.e. a excited, b absent), we would obtain using standard approximations [4] :

$$
\begin{aligned}
& \frac{\mathrm{d}}{\mathrm{d} t} \tilde{S}_{\mathrm{a}}^{-}(t)\left|\psi_{\mathrm{in}}\right\rangle= \\
& =-\tilde{S}_{\mathrm{a}}^{-}(t) \times\left(\pi \sum_{\lambda} g_{\lambda}^{2} \delta_{\mathrm{D}}\left(\omega_{\lambda}-\omega_{0}\right)\right)\left|\psi_{\mathrm{in}}\right\rangle \\
& \quad=-\frac{\Gamma}{2} \tilde{S}_{\mathrm{a}}^{-}(t)\left|\psi_{\mathrm{in}}\right\rangle
\end{aligned}
$$

where the level shift has been omitted (see App. I) ; in these expressions $\delta_{\mathrm{D}}\left(\omega_{\lambda}-\omega_{0}\right)$ is the Dirac distribution, and :

$$
\Gamma=2 \pi \sum_{\lambda} g_{\lambda}^{2} \delta_{\mathrm{D}}\left(\omega_{\lambda}-\omega_{0}\right)
$$

is the inverse of the atomic radiative lifetime : in this case, we obtain simply the radiative damping of the excited atom.

The calculations relative to the two-atoms case are detailed in Appendix I, and they yield eventually $((i, j)=(\mathrm{a}, \mathrm{b})$ or $(\mathrm{b}, \mathrm{a}))$ :

$$
\begin{aligned}
\frac{\mathrm{d}}{\mathrm{d} t} \tilde{S}_{i}^{-}(t)\left|\psi_{\mathrm{in}}\right\rangle & =-\frac{\Gamma}{2} \tilde{S}_{i}^{-}(t)\left|\psi_{\mathrm{in}}\right\rangle+ \\
+ & \frac{\Gamma}{2}\left[f\left(k_{0} \delta(t)\right)+i g\left(k_{0} \delta(t)\right)\right] \\
& \times\left(2 \tilde{S}_{i}^{+} \tilde{S}_{i}^{-}-1\right) \tilde{S}_{j}^{-}(t)\left|\psi_{\mathrm{in}}\right\rangle
\end{aligned}
$$

where $\delta(t)=\left|\mathbf{R}_{\mathrm{a}}-\mathbf{R}_{\mathrm{b}}\right|$ and $f\left(k_{0} \delta\right), g\left(k_{0} \delta\right)$ are functions depending on the orientation of the atomic dipole $\mathbf{d}_{\mathrm{eg}}$ in the molecular frame. The expressions of $f\left(k_{0} \delta\right)$ and $g\left(k_{0} \delta\right)$ are given in Appendix $\mathbf{I}$, for two cases relevant to experiments : $\Sigma$-excited 
molecular state ( $\mathbf{d}_{\mathrm{eg}}$ parallel to the internuclear axis) or, $\pi$-excited molecular state ( $\mathbf{d}_{\mathrm{eg}}$ perpendicular to the internuclear axis).

We shall see below that $f\left(k_{0} \delta\right)$ contributes to a modification of the radiative lifetime $1 / \Gamma$, while $g\left(k_{0} \delta\right)$ appears as an interaction potential between the two atoms.

The general solution of system (16) is given in Appendix II. We shall restrict ourselves here to results useful for the following, i.e. expectation values in the state $\left|\psi_{\text {in }}\right\rangle$ :

$$
\begin{aligned}
& \left\langle\tilde{S}_{i}^{+} \tilde{S}_{i}^{-}\right\rangle(\tau)=\frac{1}{2} \mathrm{e}^{-\int_{0}^{\tau} \Gamma\left(1+\varepsilon f\left(k_{0} \delta\right)\right) \mathrm{d} t} \\
& \left\langle\tilde{S}_{i}^{+} \tilde{S}_{j}^{-}\right\rangle(\tau)=\frac{\varepsilon}{2} \mathrm{e}^{-\int_{0}^{\tau} \Gamma\left(1+\varepsilon f\left(k_{0} \delta\right)\right) \mathrm{d} t} .
\end{aligned}
$$

1.4 FORCES BETWEEN THE TWO ATOMS. - By substituting equation (14) in system (11), we get (omitting $\left|\psi_{\text {in }}\right\rangle$ on the right side) :

$$
\begin{aligned}
\frac{\mathrm{d} \mathbf{P}_{i}}{\mathrm{~d} t}= & -\sum_{\lambda} g_{\lambda}^{2} \hbar \mathbf{k}_{\lambda} \tilde{S}_{i}^{+}(t) \mathrm{e}^{i \mathbf{k}_{\lambda} \cdot \mathbf{R}_{i}(t)} \times \\
& \times \int\left(\tilde{S}_{i}^{-}\left(t^{\prime}\right) \mathrm{e}^{-i \mathbf{k}_{\lambda} \cdot \mathbf{R}_{i}\left(t^{\prime}\right)}+\tilde{S}_{j}^{-}\left(t^{\prime}\right) \mathrm{e}^{-i \mathbf{k}_{\lambda} \cdot \mathbf{R}_{j}\left(t^{\prime}\right)}\right) \\
& \times \mathrm{e}^{i\left(\omega_{\lambda}-\omega_{0}\right)\left(t^{\prime}-t\right)} \mathrm{d} t^{\prime}+\text { c.c. }
\end{aligned}
$$

Using the same techniques as previously, this system can be integrated in the general case (see App. III). The results for the expectation values in the initial state $\left|\psi_{\text {in }}\right\rangle$ are :

$$
\begin{aligned}
& \frac{\mathrm{d}}{\mathrm{d} t}\left\langle\mathbf{P}_{\mathrm{a}}+\mathbf{P}_{\mathrm{b}}\right\rangle=0 \\
& \frac{\mathrm{d}}{\mathrm{d} t}\left\langle\mathbf{P}_{\mathrm{a}}-\mathbf{P}_{\mathrm{b}}\right\rangle=-\hbar \Gamma\left\langle\psi_{\mathrm{in}}\right|\left(\tilde{S}_{\mathrm{a}}^{+} \tilde{S}_{\mathrm{b}}^{-}+\tilde{S}_{\mathrm{b}}^{+} \tilde{S}_{\mathrm{a}}^{-}\right) \times \\
& \times \operatorname{grad}_{\delta}\left(g\left(k_{0} \delta\right)\right)\left|\psi_{\text {in }}\right\rangle .
\end{aligned}
$$

The first equation means that the center of mass of the pair of atoms does not experience any recoil on the average. The second equation gives the force between the two atoms. This force can be considered as deriving from a pseudo-coupling term :

$$
\left\langle V_{\mathrm{dd}}\right\rangle=\left\langle\frac{\hbar \Gamma}{2}\left(\tilde{S}_{\mathrm{a}}^{+} \tilde{S}_{\mathrm{b}}^{-}+\tilde{S}_{\mathrm{b}}^{+} \tilde{S}_{\mathrm{a}}^{-}\right) g\left(k_{0} \delta\right)\right\rangle \text {. }
$$

The sign and value of $\left\langle V_{\mathrm{dd}}\right\rangle$ for $k_{0} \delta \ll 1$ will determine the behaviour (attraction or repulsion) of the two atoms in the excited state $\left|\psi_{\text {in }}\right\rangle$. Using the values of $g\left(k_{0} \delta\right)$ calculated in Appendix I, we have :

$$
\begin{aligned}
& \left\langle\psi_{\text {in }}\left|V_{\text {dd }}\right| \psi_{\text {in }}\right\rangle_{\Pi} \cong \frac{\hbar \Gamma}{2} \varepsilon\left(\frac{3}{2} \frac{1}{\left(k_{0} \delta\right)^{3}}\right) \quad(\Pi \text {-state }) \\
& \left\langle\psi_{\text {in }}\left|V_{\text {dd }}\right| \psi_{\text {in }}\right\rangle_{\Sigma} \cong \frac{\hbar \Gamma}{2} \varepsilon\left(-3 \frac{1}{\left(k_{0} \delta\right)^{3}}\right) \quad(\Sigma \text {-state }) .
\end{aligned}
$$

Thus the $\Pi_{\mathrm{u}}(\varepsilon=+1)$ and $\Sigma_{\mathrm{g}}(\varepsilon=-1)$ states are strongly repulsive, while the $\Pi_{\mathrm{g}}$ and $\Sigma_{\mathrm{u}}$ states are attractive. This is the well-known resonant dipoledipole interaction [26].

In our experiment, $\left|\left\langle V_{\text {dd }}\right\rangle\right|$ diminishes very rapidly as $\delta$ increases (by a factor $10^{-3}$ between $10 \AA$ and $100 \AA$ ), and is less than $1 \mathrm{~cm}^{-1}$ for $\delta>100 \AA$, while the recoil energy is a few hundred $\mathrm{cm}^{-1}$. For values of $\delta$ greater than $100 \AA$, the variations of $\left\langle V_{\mathrm{dd}}\right\rangle$ are, therefore completely negligible compared to the kinetic energy; we can thus consider that after $100 \AA$ (about 10 ps after the photodissociation) the relative motion of the two atoms is uniform, and we can take as average value of $\delta=\left|\mathbf{R}_{\mathrm{a}}-\mathbf{R}_{\mathrm{b}}\right|$ the simple expression $v t$ where the recoil velocity $v$ is estimated from the available energy in the photodissociation. A spread of $v$ is due to the fact that the wavepacket associated to the relative positions of the atoms in the excited state $\left|\psi_{\text {ext }}\right\rangle$ must satisfy the Heisenberg relation $\Delta\left(R_{\mathrm{a}}-R_{\mathrm{b}}\right) \Delta\left(P_{\mathrm{a}}-P_{\mathrm{b}}\right) \geqq h$. This point will be discussed in part 2 of this paper.

1.5 Probability of Detection. - The delay between the laser pulse initiating the photodissociation and the detection time of the fluorescence photon is measured by a time-to-digital converter. The signal given by this device is the probability $p\left(\mathbf{r}, \varepsilon_{\mathrm{D}}, \mathbf{t}\right) \mathrm{d} t$ for detecting a photon polarized along $\varepsilon_{\mathrm{D}}$ at the point $\mathbf{r}$ between $t$ and $t+\mathrm{d} t$, following the photodissociation at time $t=0$ and point $\mathbf{r}=\mathbf{R}_{0}$. This probability is [5] :

$p\left(\mathbf{r}, \varepsilon_{\mathrm{D}}, t\right)=C\left\langle\psi_{\text {in }}\left|E_{\mathrm{D}}^{-}(\mathbf{r}, t) E_{\mathrm{D}}^{+}(\mathbf{r}, t)\right| \psi_{\text {in }}\right\rangle$

where $C$ is some multiplicative constant and :

$$
\begin{aligned}
E_{\mathrm{D}}^{+}(\mathbf{r}, t)\left|\psi_{\mathrm{in}}\right\rangle=i \sum_{\lambda} \sqrt{\frac{\hbar \omega_{\lambda}}{2 \varepsilon_{0} V}} & \varepsilon_{\mathrm{D}} \cdot \varepsilon_{\lambda} a_{\lambda}(t)^{i \mathbf{k}_{\lambda} \cdot \mathbf{r}}\left|\psi_{\mathrm{in}}\right\rangle .
\end{aligned}
$$

The geometry of the detection channel is shown in figure 1. The polarizer can be set parallel $\left(\varepsilon_{\mathrm{D}}=\varepsilon_{\|}\right)$ or orthogonal $\left(\varepsilon_{D}=\varepsilon_{\perp}\right)$ to the laser polarization.

Using equations (5) and (14), we obtain :

$$
\begin{array}{r}
E_{\mathrm{D}}^{+}(\mathbf{r}, t)\left|\psi_{\text {in }}\right\rangle=i \sum_{\lambda} \sqrt{\frac{\hbar \omega_{\lambda}}{2 \varepsilon_{0} V}} \varepsilon_{\mathrm{D}} \cdot \varepsilon_{\lambda} a_{\lambda}(t) \mathrm{e}^{i \mathbf{k}_{\lambda} \cdot \mathbf{r}}\left|\psi_{\text {in }}\right\rangle=i \sum_{\lambda} \sqrt{\left(\frac{\hbar \omega_{\lambda}}{2 \varepsilon_{0} V}\right)}\left(\varepsilon_{\mathrm{D}} \cdot \varepsilon_{\lambda}\right)\left(\mathbf{d}_{\mathrm{eg}} \cdot \varepsilon_{\lambda}\right) \mathrm{e}^{i\left(\mathbf{k}_{\lambda} \mathbf{r}-\omega_{\lambda} t\right)} \\
\times \int_{0}^{t}\left(\tilde{S}_{\mathrm{a}}^{-}\left(t^{\prime}\right) \mathrm{e}^{-i \mathbf{k}_{\lambda} \cdot \mathbf{R}_{\mathrm{a}}\left(t^{\prime}\right)}+\tilde{S}_{\mathrm{b}}^{-}\left(t^{\prime}\right) \mathrm{e}^{-i \mathbf{k}_{\lambda} \cdot \mathbf{R}_{\mathrm{b}}\left(t^{\prime}\right)}\right) \mathrm{e}^{i\left(\omega_{\lambda}-\omega_{0}\right) t^{\prime}} \mathrm{d} t^{\prime}\left|\psi_{\mathrm{in}}\right\rangle
\end{array}
$$


Defining $\mathbf{R}_{0}=\frac{\mathbf{R}_{\mathrm{a}}+\mathbf{R}_{\mathrm{b}}}{2}$, we have $\mathbf{R}_{\mathrm{a}}=\mathbf{R}_{0}+\frac{\boldsymbol{\delta}}{2}$ and $\mathbf{R}_{\mathrm{b}}=\mathbf{R}_{0}-\frac{\boldsymbol{\delta}}{2}$.
Using the same techniques as previously, but retaining only the farfield terms (in $\frac{1}{\left|r-\mathbf{R}_{0}\right|}$ ) we get :

$$
E_{\mathrm{D}}^{+}(r, t)\left|\psi_{\text {in }}\right\rangle=\frac{\omega_{0}^{2}\left(\mathbf{d}_{\mathrm{eg}} \cdot \varepsilon_{\mathrm{D}}\right)}{4 \pi \varepsilon_{0} c^{2}\left|\mathbf{r}-\mathbf{R}_{0}\right|} \mathrm{e}^{-i \omega_{0} \tau} \times\left(\tilde{S}_{\mathrm{a}}^{-}(\tau) \mathrm{e}^{i \frac{k_{0}}{2} \delta(\tau) \cos \theta}+\tilde{S}_{\mathrm{b}}^{-}(\tau) \mathrm{e}^{-i \frac{k_{0}}{2} \delta(\tau) \cos \theta}\right)\left|\psi_{\text {in }}\right\rangle
$$

where :

$$
\tau=t-\frac{\left|\mathbf{r}-\mathbf{R}_{0}\right|}{c}
$$

is the retarded time since the photodissociation.
Equation (22) exhibits clearly the interference between the emissions by the two dipoles which is responsible for the observed effect.

Using equation (17), we get :

$$
\begin{aligned}
& \left\langle\psi_{\mathrm{in}}\left|E_{\mathrm{D}}^{-}(\mathbf{r}, t) E_{\mathrm{D}}^{+}(\mathbf{r}, t)\right| \psi_{\mathrm{in}}\right\rangle= \\
& \quad=\left(\frac{\omega_{0}^{2}\left(\mathbf{d}_{\mathrm{eg}} \cdot \varepsilon_{\mathrm{D}}\right)}{4 \pi \varepsilon_{0} c^{2}\left|\mathbf{r}-\mathbf{R}_{0}\right|}\right)^{2} \times\left\langle\psi_{\mathrm{in}}\left|\tilde{S}_{\mathrm{a}}^{+} \tilde{S}_{\mathrm{a}}^{-}+\tilde{S}_{\mathrm{a}}^{+} \tilde{S}_{\mathrm{b}}^{-} \mathrm{e}^{-i k_{0} \delta(\tau) \cos \theta}+\tilde{S}_{\mathrm{b}}^{+} \tilde{S}_{\mathrm{a}}^{-} \mathrm{e}^{i k_{0} \delta(\tau) \cos \theta}+\tilde{S}_{\mathrm{b}}^{+} \tilde{S}_{\mathrm{b}}^{-}\right| \psi_{\text {in }}\right\rangle \\
& =\left(\frac{\omega_{0}^{2}\left(\mathbf{d}_{\mathrm{eg}} \cdot \varepsilon_{\mathrm{D}}\right)}{4 \pi \varepsilon_{0} c^{2}\left|\mathbf{r}-\mathbf{R}_{0}\right|}\right)^{2} \times\left\{1+\varepsilon \cos \left(k_{0} \delta(\tau) \cos \theta\right)\right\} \exp \left(-\int_{0}^{\tau} \Gamma\left(1+\varepsilon f\left(k_{0} \delta\left(\tau^{\prime}\right)\right) d \tau^{\prime}\right)\right) .
\end{aligned}
$$

It is convenient to define :

$$
\begin{aligned}
D_{\varepsilon}(\tau) & =\Gamma \int_{0}^{\tau}\left(1+\varepsilon f\left(k_{0} \delta\left(\tau^{\prime}\right)\right)\right) \mathrm{d} \tau^{\prime} \\
& =\Gamma \int_{0}^{\tau}\left(1+\varepsilon f\left(\Omega_{0} \tau^{\prime}\right)\right) \mathrm{d} \tau^{\prime}
\end{aligned}
$$

where $\Omega_{0}=\omega_{0} \frac{v}{c}$, and we get eventually :

$$
\begin{aligned}
& p\left(\mathbf{r}, \boldsymbol{\varepsilon}_{\mathrm{D}}, t\right)=p_{\theta \phi}(\tau)=A(\theta, \phi) \times \\
& \times\left(1+\varepsilon \cos \left(\Omega_{0} \tau \cos \theta\right)\right) \mathrm{e}^{-D_{\varepsilon}(\tau)} .
\end{aligned}
$$

This equation was the main result used in reference [1]. We have implicitly assumed here that the dispersion $\Delta\left|R_{\mathrm{a}}-R_{\mathrm{b}}\right|=\Delta(\delta)$ is much smaller than $\lambda_{0}$, otherwise the average value

$$
\left\langle\psi_{\text {in }}\left|\exp \left(i k_{0} \delta \cos \theta\right)\right| \psi_{\text {in }}\right\rangle
$$

would yield a zero result. This inequality was taken as an assumption in our simplified model ( $\$ 1.1$ ), but will be discussed in part 2 of this paper.

1.6 ANGUlar AVERAGiNG. - In the preceding sections, we calculated the probability of detection for a given orientation of the recoil axis; we must then average this result over the angular distribution of the photofragments. This approach is justified in reference [6], for the cases relevant to this paper.

In the axial recoil approximation, the recoil velocities are aligned with the molecular axis, and the angular distribution of the photofragments is simply the distribution of the molecular axis due to the excitation process. This distribution can be easily evaluated in each particular case, yielding analytical expressions for the averaged probabilities (see App. IV).

In reference [6], the situation where the excited molecular axis is isotropically distributed was also considered, and gave a better agreement with polarization measurements. Therefore we will consider the averaged probabilities for the repulsive $\Pi_{\mathrm{u}}$ and $\Sigma_{\mathrm{g}}$ states, in the case of isotropic distribution of the photofragments. Let us notice that, in our actual experimental situation, a direct excitation from the ground ${ }^{1} \Sigma_{\mathrm{g}}^{+}$state to a $\Sigma_{\mathrm{g}}$ state is impossible; nevertheless, we also consider this case for the sake of generality.

In the case of a $\Pi_{\mathrm{u}}$ excited state, equation (24) becomes, after averaging, for $\varepsilon_{\mathrm{D}}=\varepsilon_{\|}$or $\varepsilon_{\mathrm{D}}=\varepsilon_{\perp}$ :

$$
\begin{aligned}
p_{\|}^{\pi}(\tau) & =A^{\pi}\left(\frac{2}{3}+\left(\frac{1}{\Omega_{0} \tau}-\frac{1}{\left(\Omega_{0} \tau\right)^{3}}\right) \times\right. \\
\times & \left.\sin \left(\Omega_{0} \tau\right)+\frac{1}{\left(\Omega_{0} \tau\right)^{2}} \cos \left(\Omega_{0} \tau\right)\right) \\
\times & \exp \left(-\Gamma \int_{0}^{\tau}\left(1+f^{\pi}\left(\Omega_{0} \tau^{\prime}\right)\right) \mathrm{d} \tau^{\prime}\right) \quad(25 \mathrm{a}) \\
p_{\perp}^{\pi}(\tau) & =A^{\pi}\left(\frac{1}{6}-\frac{1}{\left(\Omega_{0} \tau\right)^{3}} \sin \left(\Omega_{0} \tau\right)+\frac{1}{\left(\Omega_{0} \tau\right)^{2}}\right) \times \\
& \times \exp \left(-\Gamma \int_{0}^{\tau}\left(1+f^{\pi}\left(\Omega_{0} \tau^{\prime}\right)\right) \mathrm{d} \tau^{\prime}\right) \quad(25 \mathrm{~b})
\end{aligned}
$$

where $A^{\pi}$ is a multiplicative constant. Note that for the limits $\tau \rightarrow 0$ or $\tau \rightarrow \infty$ the polarization of the 
emitted light is 0.6 , though the distribution of the photofragments is isotropic (see Refs. [6, 14]).

We can also calculate the total emission probability (in any direction with any polarization) which is obviously :

$$
\begin{aligned}
p_{\mathrm{tot}}^{\pi}(\tau)=\Gamma(1 & \left.+f^{\pi}\left(\Omega_{0} \tau\right)\right) \times \\
& \times \exp \left(-\Gamma \int_{0}^{\tau}\left(1+f^{\pi}\left(\Omega_{0} \tau^{\prime}\right)\right) \mathrm{d} \tau^{\prime}\right)
\end{aligned}
$$

such that the integral over $\tau$ of $p_{\text {tot }}^{\pi}(\tau)$ is equal to unity :

$$
\begin{aligned}
& \int_{0}^{\infty} p_{\mathrm{tot}}^{\pi}(\tau) \mathrm{d} \tau= \\
& =\left[-\exp \left(-\Gamma \int_{0}^{\tau}\left(1+f^{\pi}\left(\Omega_{0} \tau^{\prime}\right)\right)\right) \mathrm{d} \tau^{\prime}\right]_{0}^{\infty}=1 .
\end{aligned}
$$

Considering now the case of a $\Sigma_{\mathrm{g}}$ excited state, we obtain :

$$
\begin{aligned}
& p \|^{\Sigma}(\tau)=p_{\perp}^{\Sigma}(\tau)= \\
&= A^{\Sigma}\left(\frac{1}{3}-\frac{\sin \left(\Omega_{0} \tau\right)}{\left(\Omega_{0} \tau\right)^{3}}+\frac{\cos \left(\Omega_{0} \tau\right)}{\left(\Omega_{0} \tau\right)^{2}}\right) \\
& \quad \times \exp \left(-\Gamma \int_{0}^{\tau}\left(1-f^{\Sigma}\left(\Omega_{0} \tau^{\prime}\right)\right) \mathrm{d} \tau^{\prime}\right)
\end{aligned}
$$

where $A^{\Sigma}$ is a multiplicative constant. In this case the emitted light is unpolarized, with isotropic intensity. For the total emission probability, we obtain

$$
\begin{aligned}
& p_{\mathrm{tot}}^{\Sigma}(\tau)=\Gamma\left(1-f^{\Sigma}\left(\Omega_{0} \tau\right)\right) \times \\
& \times \exp \left(-\Gamma \int_{0}^{\tau}\left(1-f^{\Sigma}\left(\Omega_{0} \tau^{\prime}\right)\right) \mathrm{d} \tau^{\prime}\right) .
\end{aligned}
$$

The equations (25c) and (26b), corresponding respectively to $\Pi_{\mathrm{u}}$ and $\Sigma_{\mathrm{g}}$ excited states, make clear that the initial state is "superadiant» or « subradiant", according to the molecular parity of the state $(\varepsilon= \pm 1)$ [27]. Indeed, we obtain if $\Omega_{0} \tau \ll 1$ :

$$
\begin{aligned}
& \left.p_{\text {tot }}^{\pi} \dot{t} \tau\right) \cong 2 \Gamma \exp (-2 \Gamma \tau) \\
& p_{\text {tot }}^{\Sigma}(\tau) \cong 0
\end{aligned}
$$

The «subradiant » case corresponds to the forbidden $\Sigma_{\mathrm{g}} \rightarrow \Sigma_{\mathrm{g}}$ transition at short distance. Obviously, this selection rule does not hold any more when the distance between both atoms increases to about half a wavelength.

$p^{\pi}$ and $p^{\Sigma}$ are plotted respectively in figures $2 \mathrm{a}$ and b. Let us remark again that the main features of this curves (modulation, differences between $p_{\|}$and $\left.p_{\perp}\right)$ are due to the first bracket of equations (25) or (26), and not to the exponential term. Therefore, they can be understood as interferences between the free fields emitted coherently by the two atoms.

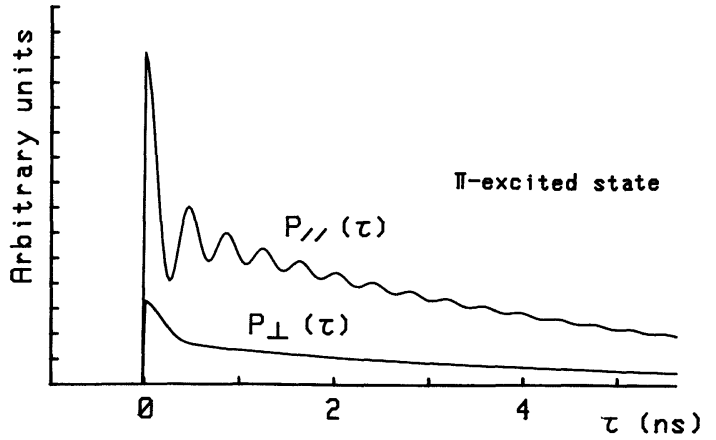

Fig. 2a. - Theoretical probabilities of detection of a fluorescence photon, with a delay $\tau$, after averaging on the molecular orientations. The atomic lifetime is $4.7 \mathrm{~ns}$, and the relative recoil velocity is $v=1000 \mathrm{~m} / \mathrm{s}$. The plotted values correspond to the excitation of a $\Pi$-state and an isotropic distribution of the photofragments.

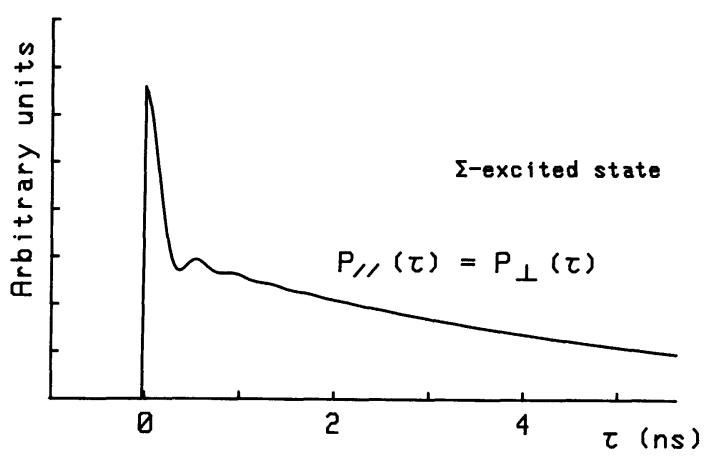

Fig. 2b. - Same quantities as (a) in similar conditions, except that the dissociating state is now a $\Sigma$-state.

Since the phase difference of the two interfering fields can be derived by considering two recoiling dipoles at a distance $\delta=v \tau$, with an initial phase lag 0 or $\pi$ according to the sign of $\varepsilon$, we see that a semi-classical picture of « moving Young slits " gives an interesting insight into the effect [1]. However, we are now to calculate the intensity correlation of the emitted light : in this case, dramatic differences between the quantum and semi-classical treatments appear.

\subsection{INTENSITY CORRELATION OF THE EMITTED} LIGHT. - In this section, we shall consider an experiment different from the previous one, but close to a proposal by Diebold [7]. The main change is that the photodissociation is now effected by a C.W. laser. In this case, photodissociations occur randomly in time, but some information can be sought in second-order properties of the intensity of the emitted light, i.e. intensity correlations or equivalently noise-power spectrum of the photoelectric current. For instance, due to the modulation at $\Omega_{0}$ of the emitted intensity (Fig. 2), one could hope to find the «beat note » at $\Omega_{0}$ in the noise power spectrum of the detected 
photocurrent [7]. However, it was recognized that such a modulation would not exist, due to the singlephoton character of the dissociation process $[20,22]$. In this part, we want to elaborate further on this point : in our approach, we can show that the singlephoton modulation actually does not exist, but that a modulation can nevertheless be observed, due to contributions from different molecules to the emitted light. However, stringent conditions about the spatial coherence of the source will appear, which are very far from being satisfied in our experimental configuration.

To be more quantitative, let us recall that a spectrum analyzer yields the Fourier transform of the intensity correlation of the detected light ; this latter quantity, which can be also directly measured with a time-to-digital converter, is the joint probability density $P_{12}\left(t_{1}, t_{2}\right)$ for detecting two photons with a time delay $\left(t_{2}-t_{1}\right)$ (this joint probability does not depend on $\left(t_{1}+t_{2}\right)$ for stationary light). One shows that [5] :

$$
P_{12}\left(t_{1}, t_{2}\right)=\int_{S_{1}} \mathrm{~d} \mathbf{r}_{1} \int_{S_{2}} \mathrm{~d} \mathbf{r}_{2} p_{12}\left(\mathbf{r}_{1}, t_{1}, \mathbf{r}_{2}, t_{2}\right)
$$

where :

$$
\begin{aligned}
& p_{12}\left(\mathbf{r}_{1}, t_{1}, \mathbf{r}_{2}, t_{2}\right)= \\
& \quad=\left\langle E_{\mathrm{D}}^{-}\left(\mathbf{r}_{1}, t_{1}\right) E_{\mathrm{D}}^{-}\left(\mathbf{r}_{2}, t_{2}\right) E_{\mathrm{D}}^{+}\left(\mathbf{r}_{2}, t_{2}\right) E_{\mathrm{D}}^{+}\left(\mathbf{r}_{1}, t_{1}\right)\right\rangle .
\end{aligned}
$$

The integrals over $\mathbf{r}_{1}$ and $\mathbf{r}_{2}$ are taken over the detecting area $S_{1}$ and $S_{2}$ of the photomultipliers PM1 and PM2 (Fig. 3). We shall assume that these detecting areas are images of each other in the beamsplitter BS, such that $S_{1}=S_{2}=S$.
$E_{\mathrm{D}}(\mathbf{r}, t)$ is given as a sum over the emitting pairs of atoms, labelled by $k$ [8] :

$$
E_{\mathrm{D}}^{+}(\mathbf{r}, t)=\sum_{k} E_{\mathrm{D} k}^{+}(\mathbf{r}, t) .
$$

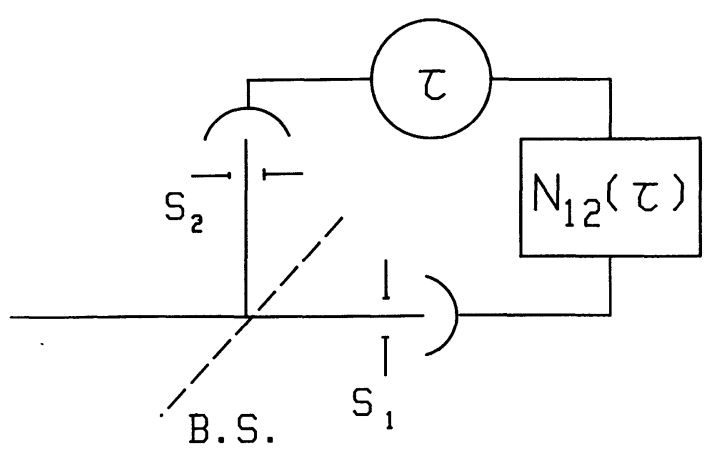

Fig. 3. - Possible intensity correlation set-up for the measurement of the joint probability density $P_{12}\left(t_{1}, t_{2}\right)$. The experimental quantity is the coincidence rate $N_{12}(\tau)$ as a function of $\tau=t_{2}-t_{1}$. The detection areas $S_{1}$ and $S_{2}$ are images of each other through the beamsplitter B.S.

$E_{\mathrm{D} k}^{+}(\mathbf{r}, t)$ is the contribution of an individual pair of atoms ; assuming that a photodissociation occurs at time $t_{k}$ and point $\mathbf{R}_{k}, E_{\mathrm{D} k}^{+}(\mathbf{r}, t)$ is given by an expression similar to equation (22), in which $\mathbf{R}_{0}$ is changed into $\mathbf{R}_{k}$ and $\tau$ into $\left(\tau-t_{k}\right)$.

$p_{12}\left(\mathbf{r}_{1}, t_{1}, \mathbf{r}_{2}, t_{2}\right)$ thus appears as a quadruple sum, which must be averaged over the random position $\mathbf{R}_{k}$ and time $t_{k}$. Due to the random phases of the different emitters, this sum can be written [4] :

$$
\begin{aligned}
p_{12}\left(\mathbf{r}_{1}, t_{1}, \mathbf{r}_{2}, t_{2}\right) & =\sum_{k}\left\langle E_{\overline{\mathrm{D} k}}^{-}\left(\mathbf{r}_{1}, t_{1}\right) E_{\overline{\mathrm{D}} k}^{-}\left(\mathbf{r}_{2}, t_{2}\right) E_{\mathrm{D} k}^{+}\left(\mathbf{r}_{2}, t_{2}\right) E_{\mathrm{D} k}^{+}\left(\mathbf{r}_{1}, t_{1}\right)\right\rangle+\sum_{l \neq m}\left\{\left\langle E_{\overline{\mathrm{D} l}}^{-}\left(\mathbf{r}_{1}, t_{1}\right) E_{\mathrm{D} l}^{+}\left(\mathbf{r}_{1}, t_{1}\right)\right\rangle \times\right. \\
& \left.\times\left\langle E_{\mathrm{D} m}^{-}\left(\mathbf{r}_{2}, t_{2}\right) E_{\mathrm{D} m}^{+}\left(\mathbf{r}_{2}, t_{2}\right)\right\rangle+\left\langle E_{\overline{\mathrm{D} l}}^{-}\left(\mathbf{r}_{1}, t_{1}\right) E_{\mathrm{D} l}^{+}\left(\mathbf{r}_{2}, t_{2}\right)\right\rangle\left\langle E_{\mathrm{D} m}^{-}\left(\mathbf{r}_{2}, t_{2}\right) E_{\mathrm{D} m}^{+}\left(\mathbf{r}_{1}, t_{1}\right)\right\rangle\right\}
\end{aligned}
$$

In a semi-classical theory, i.e. with a non-quantized field the first term of this sum can be written as an autocorrelation function of the emitted intensity, and is modulated if the intensity is modulated. Here the result is completely different since $E_{\mathrm{D} k}^{+}\left(r_{2}, t_{2}\right)$ $E_{\mathrm{D} k}^{+}\left(r_{1}, t_{1}\right)\left|\psi_{i}\right\rangle=0$ (see Eq. (22); one molecule emits only one photon [20], which can only be detected once.

Thus the contribution of the first term in equation (28) in zero. After averaging over $t_{k}$, the second term appears to be constant (independent of $\left.\left(t_{1}-t_{2}\right)\right)$ : it is the usual « accidental coincidences » term of photon counting experiments, and we denote it $C_{12}$.

The third term depends on $\left(t_{1}-t_{2}\right)$ and also on $\left(\mathbf{r}_{1}-\mathbf{r}_{2}\right)$; it is convenient to introduce the nor- malized first-order coherence $g^{(1)}\left(\mathbf{r}_{1}-\mathbf{r}_{2}, t_{1}-t_{2}\right)$ [4], and we obtain :

$$
\begin{aligned}
& p_{12}\left(\mathbf{r}_{1}, t_{1}, \mathbf{r}_{2}, t_{2}\right)= \\
& \quad=C_{12}\left(1+\left|g^{(1)}\left(\mathbf{r}_{1}-\mathbf{r}_{2}, t_{1}-t_{2}\right)\right|^{2}\right) .
\end{aligned}
$$

To proceed further, let us recall some basic properties of the function $\left|g^{(1)}\left(\mathbf{r}_{1}-\mathbf{r}_{2}, t_{1}-t_{2}\right)\right|$. This function has non-negligible value in an area $\sigma_{c}$ around $\left(\mathbf{r}_{1}-\mathbf{r}_{2}\right)=\mathbf{0}$, and in a time interval $\tau_{c}$ around $\left(t_{1}-t_{2}\right)=0$. The quantities $\sigma_{c}$ and $\tau_{c}$ are respectively the coherence area and the coherence time of the emitted light. From equation (28) and (29), we see that $\left|g^{(1)}(0,0)\right|=1$; the function $\left|g^{(1)}\left(\mathbf{r}_{1}-\mathbf{r}_{2}, t_{1}-t_{2}\right)\right|$ is therefore decreasing from 1 to 0 as $\left|\mathbf{r}_{1}-\mathbf{r}_{2}\right|$ or $\left|t_{1}-t_{2}\right|$ increase. 
Eventually, let us notice that $\left|g^{(1)}\left(0, t_{1}-t_{2}\right)\right|$ is nothing but the Fourier transform of the frequency spectrum of the emitted light ; when several spectral components are involved, some modulations may be expected during the coherence time $\tau_{\mathrm{c}}$.

However, the visibility of these modulations will depend on spatial coherence conditions. Indeed, let us now calculate the measured quantity $P_{12}\left(t_{1}\right.$, $t_{2}$ ) given by equation (27). Assuming $\sigma_{\mathrm{c}} \ll S$, the spatial integration over $\mathbf{r}_{1}$ and $\mathbf{r}_{2}$ yields :

$P_{12}\left(t_{1}, t_{2}\right)=C_{12} S^{2}\left(1+\frac{\sigma_{\mathrm{c}}}{S}\left|g^{(1)}\left(0, t_{1}-t_{2}\right)\right|^{2}\right)$.

In the opposite case $S \ll \sigma_{\mathrm{c}}$, we would get :

$$
P_{12}\left(t_{1}, t_{2}\right)=C_{12} S^{2}\left(1+\left|g^{(1)}\left(\mathbf{0}, t_{1}-t_{2}\right)\right|^{2}\right) \text {. }
$$

Using the relations

$$
g^{(1)}(0,0)=1 \text { and } g^{(1)}(0, \infty)=0,
$$

we have :

$$
P_{12}(t, t)=2 P_{12}(t, t+\infty) .
$$

This is the well-known Hanbury-Brown and Twiss effect. In this case, some modulations may be expected during the decrease of $\left|g^{(1)}\left(0, t_{1}-t_{2}\right)\right|^{2}$ from 1 to 0 ; note that some care must be taken to observe such modulations, since $\left|g^{(1)}\left(0, t_{1}-t_{2}\right)\right|$ is sensitive to the inhomogeneous linewidth of the transition, via the coherence time $\tau_{\mathrm{c}}$.

However, if $\sigma_{\mathrm{c}} \ll S$, the visibility of the modulation is at best $\sigma_{\mathrm{c}} / S \ll 1$ (see Eq. (30)). In this case, the modulation likely to be overwhelmed by counting noise; a quantitative discussion relevant to our experimental situation will be given in part 3 .

\section{Molecular physics discussion.}

2.1 PReVious WORKS ABOUT MOLECULAR STATES OF $\mathrm{Ca}_{2}$. - A general quantum mechanical treatment of photodissociation by a C.W. laser has been presented in [6]. However, the interference between the fields radiated by the two atoms was not taken into account then, and such a treatment should be quite intricate for describing the present experiment. Therefore, our aim here is to justify the initial state $\left|\psi_{\text {in }}\right\rangle$ used in the previous section. Moreover, we shall study more precisely the recoil speed distribution of the calcium atoms after the excitation by the laser pulse.

In [6], the knowledge concerning the electronic states of $\mathrm{Ca}_{2}$ was summarized. Some new results have been obtained since, concerning mainly the $\mathrm{A}$ and $B{ }^{1} \Sigma_{u}^{+}$states [9-11]. In fact we are mainly interested in the $\mathrm{X}^{1} \Sigma_{\mathrm{g}}^{+}$ground state, well known thanks to the work of Vidal [12], and in the

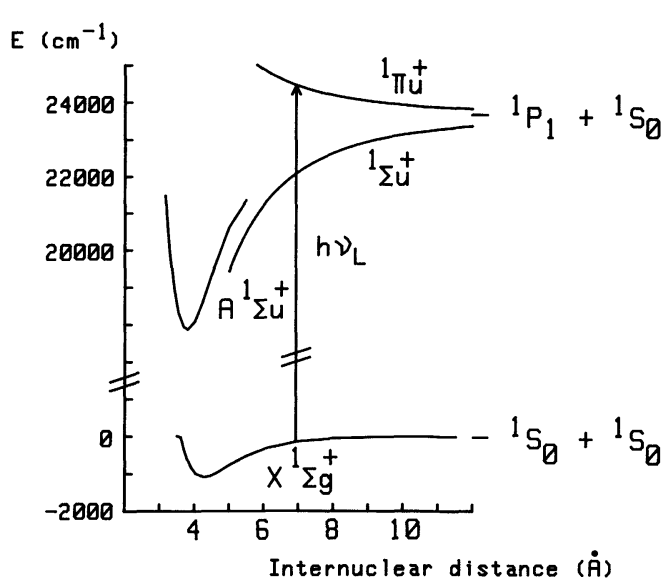

Fig. 4. - Potential curves for the $\mathrm{X}^{1} \Sigma_{\mathrm{g}}^{+}, \mathrm{A}^{1} \Sigma_{\mathrm{u}}^{+},{ }^{1} \Sigma_{\mathrm{u}}^{+}$and ${ }^{1} \Pi_{\mathrm{u}}$ states discussed in the text. The arrow corresponds to the vertical transition with an energy equal to the energy of the laser photons (at $406.7 \mathrm{~nm}$ ). This graphical representation clearly exhibits the fact that the experiment involves only the relatively long range part of the $X$ and ${ }^{1} \Pi_{u}$ potential curves.

${ }^{1} \Pi_{\mathrm{u}}$ state dissociating in $\mathrm{Ca}\left({ }^{1} \mathrm{~S}_{0}\right)+\mathrm{Ca}\left({ }^{1} \mathrm{P}_{1}\right)$, already known through an experiment performed on $\mathrm{Ca}_{2}$ trapped in rare gas matrices [13].

Our previous results $[6,14]$ concerning the polarization of the $\mathrm{Ca}$ atomic fluorescence $(422.7 \mathrm{~nm})$ excited by photodissociation of $\mathrm{Ca}_{2}$ molecules by a C.W. laser at $406.7 \mathrm{~nm}$ have shown unambiguously that the molecular excited state involved is this ${ }^{1} \Pi_{u}$ state (Fig. 4). For large value of the internuclear distance [15], the part of this state corresponding to internal degrees of freedom is well approximated by a symmetrized product of atomic wavefunctions for atoms a and b (see Eq. (10)) :

$$
\left|{ }^{1} \Pi_{\mathrm{u}}\right\rangle_{\delta \rightarrow \infty}=\frac{1}{\sqrt{2}}\left\{|\mathrm{~g}\rangle_{\mathrm{a}}|\mathrm{e}\rangle_{\mathrm{b}}+|\mathrm{e}\rangle_{\mathrm{a}}|\mathrm{g}\rangle_{\mathrm{b}}\right\}
$$

where $|\mathrm{g}\rangle$ is the $4 \mathrm{~s}^{2}{ }^{1} \mathrm{~S}_{0}$ atomic ground state, and |e) a coherent superposition of the $m_{J}= \pm 1$ sublevels of the excited $4 s 4 p{ }^{1} P_{1}$ state $[6,14]$.

2.2 EXTERNAL DEGREES OF FREEDOM. - The wavepacket describing the relative motion of the two atoms is essentially a superposition of levels of the continuum of the ${ }^{1} \Pi_{u}$ state, depending on the dynamics of the photodissociation process. For the nearly Fourier-transform pulses used in our experiment, a reasonable assumption for the initial dispersion of the relative position $\Delta\left|R_{\mathrm{a}}-R_{\mathrm{b}}\right|$ is the distance $\left(v \tau_{\mathrm{L}}\right)$, where $v$ is the recoil velocity and $\tau_{\mathrm{L}}$ the pulse duration. Correspondingly, one shows easily that :

$$
\Delta\left|P_{\mathrm{a}}-P_{\mathrm{b}}\right| \sim \frac{\hbar \omega_{0}}{\mathrm{c}} \frac{(\delta \omega)_{\mathrm{L}}}{\Omega_{0}}
$$

where $\tau_{\mathrm{L}}(\delta \omega)_{\mathrm{L}} \sim 1$, and $\Omega_{0}=\omega_{0} \frac{v}{c}$. 
We have assumed in part 1 that $\Delta\left|R_{\mathrm{a}}-R_{\mathrm{b}}\right|$ was much smaller that $\lambda_{0}$. One can easily check that equation (32) gives in that case $\Delta\left|P_{\mathrm{a}}-P_{\mathrm{b}}\right| \gg$ $h / \lambda_{0}$; our hypothesis are therefore consistent with the Heisenberg relation

$$
\Delta\left|R_{\mathrm{a}}-R_{\mathrm{b}}\right| \quad \Delta\left|P_{\mathrm{a}}-P_{\mathrm{b}}\right| \geqq \hbar .
$$

There is also an upper limit on $\Delta\left|P_{\mathrm{a}}-P_{\mathrm{b}}\right|$, in order not to wash out the modulation by an excessive velocity spread; the corresponding limit on $(\delta \omega)_{\mathrm{L}}$ is $(\delta \omega)_{\mathrm{L}} \ll\left(\omega_{\mathrm{L}}-\omega_{0}\right)$. It is clearly satisfied in our experiment.

Eventually, a practical limiting factor to be considered is the velocity spread, due to the initial distribution in the rovibrational levels of the X-state. This point is discussed in the next sections, and we shall obtain both a mean value and a r.m.s. deviation for the recoil velocities in the actual experiment.

2.3 ExCITATION PROCESS. - During their flight across the laser beam, the molecules are irradiated by a train of a few (up to 5 or 6 ) pulses, with a pulse peak power about $60 \mathrm{~kW} / \mathrm{cm}^{2}$. Since the time between pulses (12 ns) is much longer than the times relevant to the experiment (a few ns), the effects of the various pulses add incoherently. We can describe this excitation process by an excitation rate $\gamma$ $\left(v^{\prime \prime}, J^{\prime \prime}, M^{\prime \prime}\right)$ as long as the Rabi frequency describing the $\mathrm{X}^{1} \Sigma_{\mathrm{g}}^{+}-{ }^{1} \Pi_{\mathrm{u}}$ coupling is not too large; this condition is satisfied here as discussed in reference [6]. The excitation rate of the level $X\left(v^{\prime \prime}, J^{\prime \prime}\right.$, $\left.M^{\prime \prime}\right)$ is :

$$
\begin{aligned}
& \gamma\left(v^{\prime \prime} J^{\prime \prime} M^{\prime \prime}\right)= \\
& \quad=\frac{2 \Pi}{\hbar} \sum_{J M}\left|\left\langle X\left(v^{\prime \prime} J^{\prime \prime} M^{\prime \prime}\right)\left|\mathbf{D} \cdot \mathbf{E}_{\mathrm{L}}\right|^{1} \Pi_{\mathrm{u}} \varepsilon_{\mathrm{c}} J M\right\rangle\right|^{2}
\end{aligned}
$$

where $\mathbf{D} \cdot \mathbf{E}_{\mathrm{L}}$ is the molecule-laser field coupling, and the energies $\varepsilon_{\mathrm{c}}$ (continuum level) and $E_{v^{\prime \prime} J^{\prime \prime} M^{\prime \prime}}$ (ground level) satisfy $\varepsilon_{\mathrm{c}}=E_{v^{\prime \prime} J^{\prime \prime} M^{\prime \prime}}+h \nu_{\mathrm{L}}$. This excitation rate is a function of $M^{\prime \prime}$. After averaging over $M^{\prime \prime}$, in the high $J^{\prime \prime}$ limit, one gets :

$$
\gamma\left(v^{\prime \prime} J^{\prime \prime}\right)=\frac{2 \Pi}{3 \hbar} E_{\mathrm{L}}^{2}\left|\left\langle\mathrm{X}\left(v^{\prime \prime} J^{\prime \prime}\right)\left|D_{1}^{1}\right|^{1} \Pi_{\mathrm{u}} \varepsilon\right\rangle\right|^{2} .
$$

This expression involves the Franck-Condon density (FCD) $\left|\left\langle\mathrm{X}\left(v^{\prime \prime} J^{\prime \prime}\right) \mid{ }^{1} \Pi_{\mathrm{u}} \varepsilon\right\rangle\right|^{2}$ (the matrix element of the dipole is assumed to be independant of $\delta$ in the important range of internuclear distance). We have calculated the FCD, using the approximation formula given by Child [16], which seems sufficient here.

In figure 5 the FCD is plotted as a function of the energy of the level $\mathrm{X}\left(v^{\prime \prime} J^{\prime \prime}\right)$, for some selected values of $J^{\prime \prime}$. Using the molecular dipole moment deduced from the atomic value, we can obtain the excitation rate corresponding to the maximum value of the FCD :

$$
\gamma_{\max } \sim 6 \times 10^{10} \mathrm{~s}^{-1} .
$$

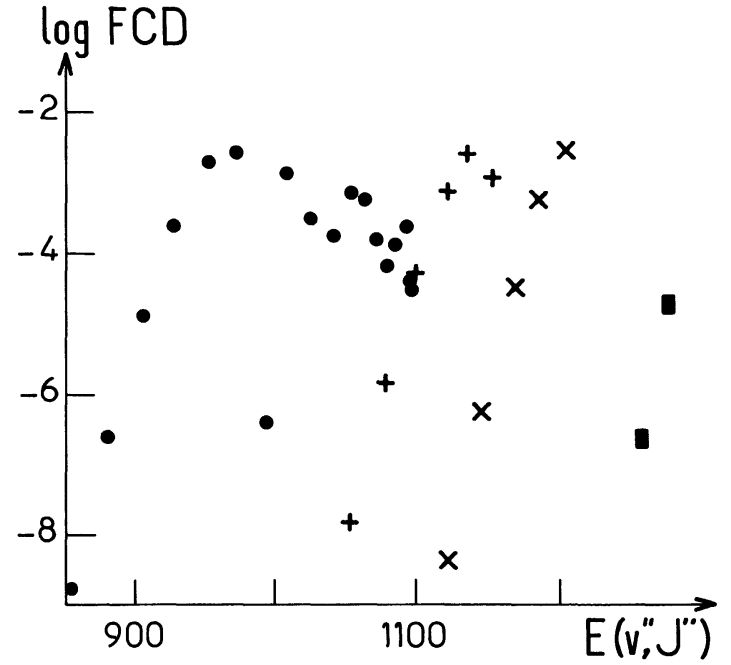

Fig. 5. - Plot of $\log _{10}$ FCD (in $\mathrm{cm}$ ) as a function of the energy of the state $\mathrm{X}\left(v^{\prime \prime} J^{\prime \prime}\right)$ for various values of $J^{\prime \prime}\left(\bullet J^{\prime \prime}=0,+J^{\prime \prime}=100, \times J^{\prime \prime}=118, \square J^{\prime \prime}=141\right)$. The spread of the data for $J^{\prime \prime}=0$ is explained by the fact that the FCD is a rapidly oscillating function of $\mathrm{E}$ for low $J^{\prime \prime}$ values.

As the molecules are submitted to several pulses of $120 \mathrm{ps}$ each, the number of excited molecules, at the maximum of the FCD, is not linear as a function of the laser power ; however, the linear regime may be considered as a reasonable approximation, since it applies to most of the rovibrational levels. In this approximation, the photodissociation signal due to the level $\mathrm{X}\left(v^{\prime \prime} J^{\prime \prime}\right)$ is simply proportionnal to the product of the population of this level $\mathrm{P}\left(v^{\prime \prime} J^{\prime \prime}\right)$ by the corresponding FCD. It is then easy to calculate the repartition of recoil energies if we know $\mathrm{P}\left(v^{\prime \prime} J^{\prime \prime}\right)$. The next paragraph discusses this point.

2. $4 \mathrm{Ca}_{2}$ MOLECULAR BEAM. - Our beam works in a supersonic regime. The source pressure $p_{0}$ and the oven diameter $D$ are sufficient $(D \cong 0.5 \mathrm{~mm}$; $p_{0}=67 \mathrm{mbar}$ for $\left.T \sim 1400 \mathrm{~K}\right)$. Moreover, MeiwesBroer [17] has produced and characterized a supersonic Calcium beam in similar conditions $(D \cong$ $0.8 \mathrm{~mm} ; p_{0} \cong 12 \mathrm{mbar}$ for $\left.T \sim 1250 \mathrm{~K}\right)$. He has measured the translational temperature by time of flight analysis, and the rovibrational temperature of low lying levels by laser induced fluorescence, with the same result $T=150 \mathrm{~K}$.

Unfortunately, these measurements are not sensitive to the population of the states close to the dissociation limit, which are responsible for our photodissociation process. Usually, in a supersonic expansion the relaxation is not complete and the population of highly excited rovibrational levels is not described by the temperature measured for the first levels. Moreover, we are going to see that high $J^{\prime \prime}$ quasibound levels, above the dissociation limit of the $J^{\prime \prime}=0$ ground state, seem to contribute to the 
observed signal ; such states can be created by 3 body collisions in the first stages of the expansion.

The lifetime of these states can be estimated by a simple tunnel effect model, involving the energy of the state and the "thickness" of the rotational barrier. We shall assume that a given quasibound level will contribute if its lifetime is greater than the time of flight between the aperture of the oven and the interaction region $(0.1 \mathrm{~ms})$.

In any case, the FCD is important only for levels at less than $205 \mathrm{~cm}^{-1}$ from the dissociation limit ; a reasonable assumption is thus to take the population $\mathrm{P}\left(v^{\prime \prime} J^{\prime \prime}\right)$ proportional to the statistical weight $\left(2 J^{\prime \prime}+1\right)$.

2.5 RECOIL ENERGY DISTRIBUTION. - To summarize previous discussions, the contribution of an sufficiently bound level $X\left(v^{\prime \prime} J^{\prime \prime}\right)$ to the signal is taken proportional to the product of the FCD with $\left(2 J^{\prime \prime}+1\right)$; knowing the energy of this level, it is then easy to calculate the recoil energy distribution. The result is plotted in figure 6 ; we have summed the contributions of all the levels inside a given $10 \mathrm{~cm}^{-1}$ interval.

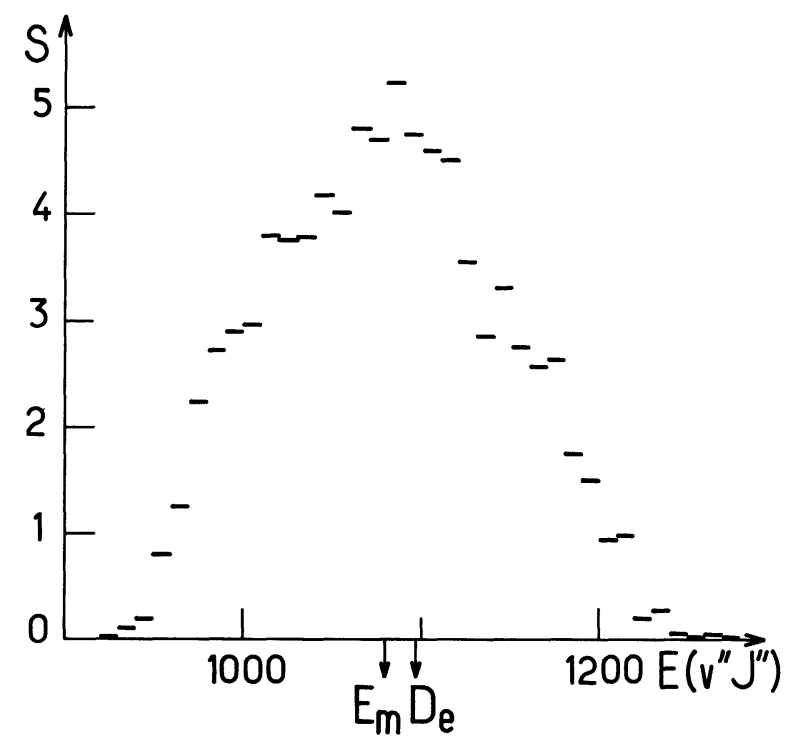

Fig. 6. - Photodissociation signal as a function of the energy $E\left(v^{\prime \prime} J^{\prime \prime}\right)$ of a level $X\left(v^{\prime \prime} J^{\prime \prime}\right)$; each horizontal bar indicate the contribution of a given $10 \mathrm{~cm}^{-1}$ interval. The mean energy $E_{\mathrm{m}}$ of this distribution is very close to the dissociation limit $D_{\mathrm{e}}$, due to the contribution of quasibounds levels.

We see that levels above the dissociation limit $D_{\mathrm{e}}$ contribute appreciably to the signal, and the mean energy $E_{\mathrm{m}}$ of the contributing levels is very close to $D_{\mathrm{e}}$.

The average recoil energy is :

$$
\bar{E}_{\mathrm{r}}=912 \mathrm{~cm}^{-1}
$$

and its r.m.s. deviation is small :

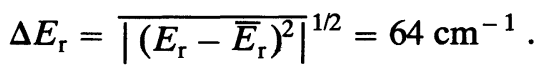

The corresponding recoil speed and r.m.s. deviation are :

$$
\bar{v}=1045( \pm 40) \mathrm{ms}^{-1} .
$$

The value deduced from the beat frequency is :

$$
v_{\text {exp }}=1100 \pm 70 \mathrm{~ms}^{-1}
$$

which is in agreement with the calculated value within one standard deviation. If quasibound levels were not taken into account, the calculated value would be $1020( \pm 20) \mathrm{ms}^{-1}$, increasing the discrepancy to more than one standard deviation.

Though this difference is not fully conclusive, this result gives an indication that quasibound states cannot be excluded a priori when levels close to the dissociation limit are involved.

Finally, let us notice that the Franck-Condon density has an effect on the transition probability strong enough to select only a narrow range of velocities $(\Delta \bar{v} / \bar{v}<5 \%)$; such a circumstance is very favourable from the point of view of our experiment.

\section{Discussion of the $\mathrm{Ca}_{2}$ experiment.}

3.1 EXPERIMENTAL SET-UP. - The experimental set-up, already described elsewhere [1], is sketched in figure 7. A molecular beam of calcium is irradiated by the $406.7 \mathrm{~nm}$ violet line of a mode-locked krypton ion laser (Spectra-Physics 171-01). Mode-locking is achieved by standard techniques (intracavity acousto-optical modulation) and yields pulses with $120 \mathrm{ps}$ full width half maximum, at a repetition rate $82.3 \mathrm{MHz}$. The average power is $60 \mathrm{~mW}$.

The laser beam is focused at the interaction region (beam waist radius at $1 / e^{2} \cong 30 \mu \mathrm{m}$ ). The fluores-

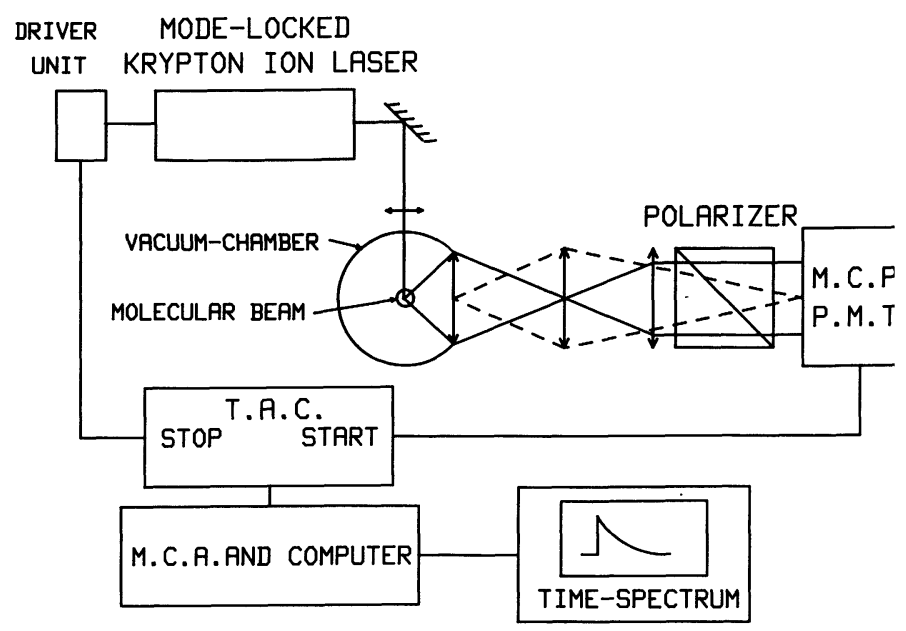

Fig. 7. - Experimental set-up (see text). 
cent light is collected by a wide aperture aspherical lens $(f / 0.8)$ with its axis in a direction perpendicular to both the atomic beam and the laser polarization (see Fig. 1); the lens is followed by an interference filter centred at $422.7 \mathrm{~nm}$ (2 nm FWHM), a polarizer with an orientation // or $\perp$ (Fig. 1), and the detector.

The overall detection efficiency of the detection channel is about $10^{-3}$. In order to achieve a sufficient time resolution, we have used a micro-channel photomultiplier tube (Hamamatsu R 1645 U or ITL CPP M3) ; the transit-time-spread of these tubes, which limites intrinsically the time resolution in the single photoelectron counting mode, is about 100 ps (full width at half maximum).

The pulse from the M.C.P. PMT is shaped by a constant-fraction discriminator, then it feeds the START input of a time-to-digital converter. When a start pulse is present, a NIM logic module allows a pulse synchronous with the radio frequency of the mode-locker to be fed to the STOP input of the T.D.C. After accumulation of the data in the memory of a computer acting as a multichannel analyser, we obtain a time-spectrum with 50 ps per channel.

The overall resolution of the system has been measured by allowing air to fill the vacuum chamber. The Rayleigh scattering of the mode-locked laser light by air at about atmospheric pressure is sufficient to yield a time-spectrum of the laser pulse (Fig. 8). Some care had to be taken to avoid extra peaks, due to stray reflections onto some parts of the optics. A gaussian curve with 180 ps full width at half maximum can be fitted onto the laser pulse time-spectrum. It renders an account of the time resolution of the whole experimental system. Theoretical timespectrum for the fluorescence of $\mathrm{Ca}_{2}$ molecules are then derived by convoluating the formulas of $\$ 1.6$

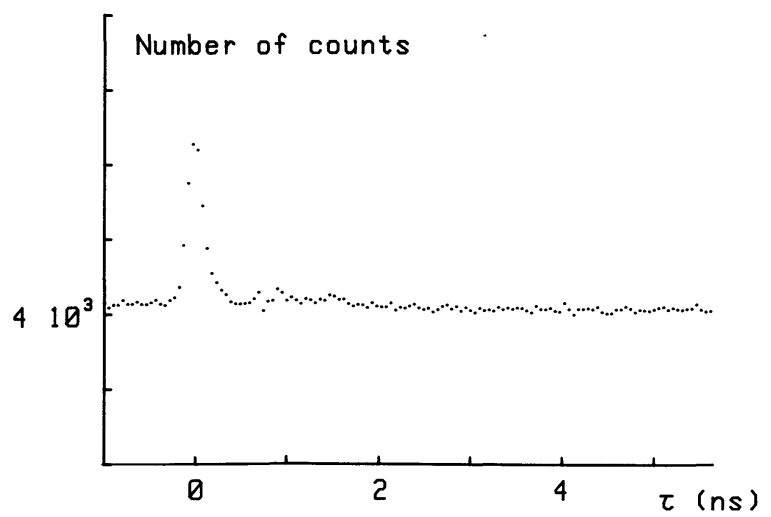

Fig. 8. - Laser pulse time-spectrum obtained from Rayleigh scattering of the laser light by air at atmospheric pressure (50 ps per channel). The width of the signal (180 ps FWHM), includes contributions from the laser pulse $(120 \mathrm{ps})$, the photomultiplier $(100 \mathrm{ps})$ and the electronics $(80 \mathrm{ps})$. by the laser pulse time-spectrum. Three parameters remain free for the final fitting:

(i) The time origin.

(ii) The flat background due to dark counts of the PMT.

(iii) The modulation frequency $\Omega_{0} / 2 \pi$.

3.2 Numerical FITs. - The result of this fitting procedure are shown in figure 9 . The agreement for the // polarization is good, yielding the value of the modulation period :

$$
\frac{2 \pi}{\Omega_{0}}=385 \pm 25 \mathrm{ps} \text {. }
$$
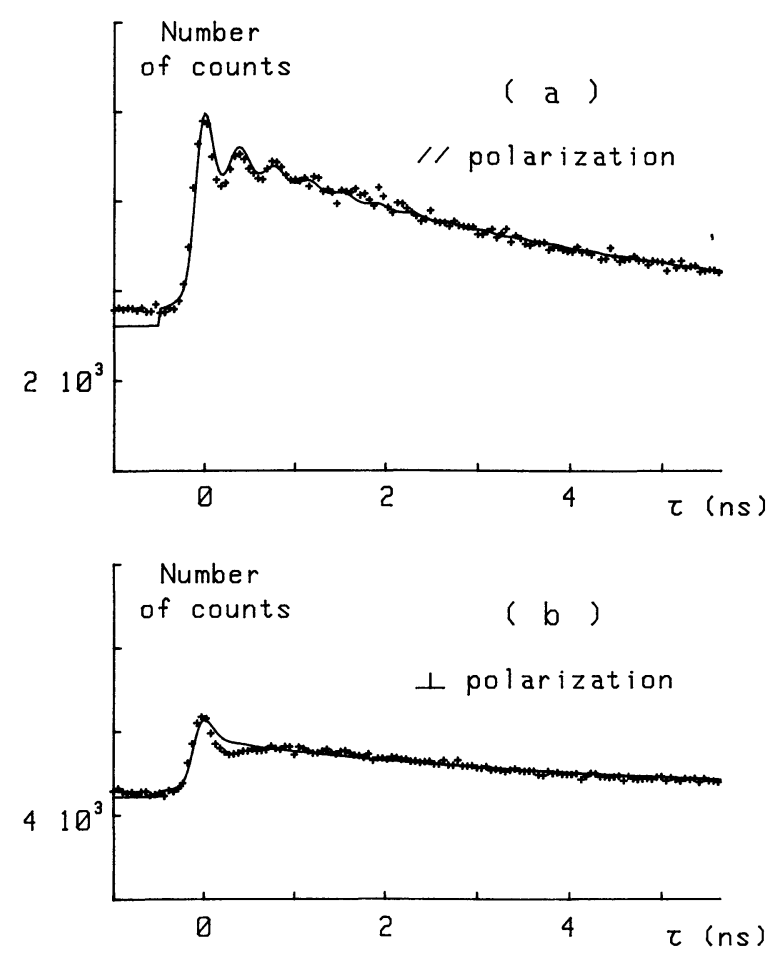

Fig. 9. - Experimental data and fitted theoretical curves for parallel (a) and perpendicular (b) polarization configurations. A discrepancy appears mainly on $\mathrm{p}_{\perp}$, as a hole around $\tau=400 \mathrm{ps}$ (50 ps per channel).

However, a discrepancy appears clearly for the $\perp$ polarization. In order to explain this discrepancy, one must notice that any phenomenon which creates an extra anisotropy among the angular distribution of the molecular axis can produce effects of this type. We consider such phenomena in the following, and discuss their relevance to the observed discrepancy.

(i) Molecular rotation between excitation and dissociation. - This effect has been considered in detail in [6]. It was shown to be small in our experimental situation (overall rotation: less than one radian). Moreover, the first effect of such a molecular rotation would be a depolarization of the 
fluorescence light, which was not observed in the experiment.

(ii) Failure of the hypothesis leading to equation (25). - Equation (25) have been obtained assuming an isotropic distribution of recoil axis. However, the other cases treated in Appendix IV have been considered, without reducing appreciably the discrepancy.

(iii) Finite detection solid angle. - Some estimations have been made to take into account the collection solid angle $(f / 0.8$ lens $)$. The depolarization due to this wide angle appear to be small (less than $3 \%$, see Ref. [6]), and the corresponding correction on $p_{\|}$or $p_{\perp}$ are not sufficient to get a better fit.

(iv) Resonant reabsorption at $\lambda_{0}=422.7 \mathrm{~nm}$. The on-resonance optical thickness of the $0.5 \mathrm{~mm}$ atomic beam at a density about $5 \times 10^{11}$ atoms $/ \mathrm{cm}^{3}$ is much greater than one. But reabsorption of the emitted light is mainly determined by Doppler effect. We have seen that the velocity of the dissociated atoms relative to the centre of mass of the molecule is about $500 \mathrm{~m} / \mathrm{s}$. Hence the fluorescence light emitted towards the detection axis $\mathrm{O} z$ (Fig. 1) is scattered if $\theta$ is close to $\pi / 2$, while it is sufficiently Doppler-shifted to go across the atomic beam if $\theta$ is close to 0 . The design of the detection channel makes that only a small fraction of the
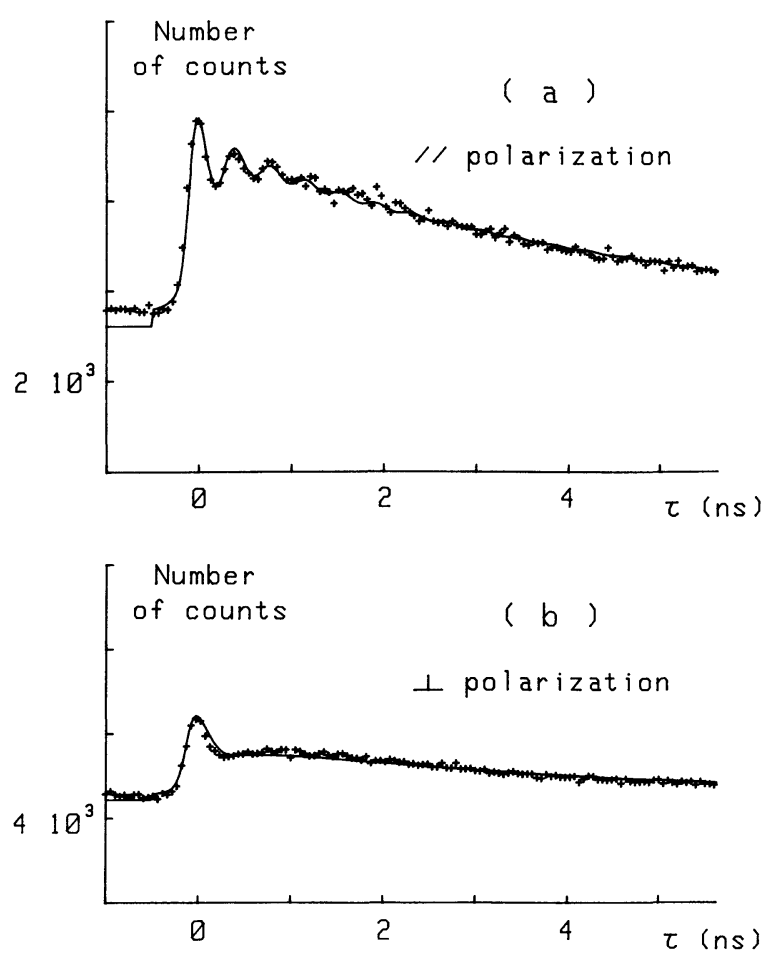

Fig. 10. - A better agreement is obtained by including some effects of atomic resonant absorption in the calculations (compare with Fig. 9). scattered light is detected [18], so that the net result is a loss of fluorescence light for molecules with $\theta$ close 0 .

The exact calculation of the modified angular distribution resulting from this effect is very difficult to handle ; we have taken it into account approximatively by "digging " a « hole » in the angular distribution for $\theta$ close to $\pi / 2$. We see on figure 10 that this procedure was fairly succcessful. The previous discrepancy is thus attributed mainly to resonant scattering, though other contributions cannot be excluded.

3.3 SPATIAl COHERENCE OF THE SOURCE. - We have seen in $\S 1.7$ that the visibility of the modulation in a C.W. experiment would be of the order $\sigma_{c} / S$, where $\sigma_{c}$ is the coherence area of the source and $S$ the surface of the illuminated part of the photocathode. However, this quantity is more easily evaluated at the source : if the size of the source is $d$, and the solid angle of collection is $\boldsymbol{\Omega}_{\mathrm{c}}$, we have :

$$
\frac{\sigma_{\mathrm{c}}}{S} \sim \frac{\lambda_{0}^{2}}{d^{2} \Omega_{\mathrm{c}}} .
$$

Typical orders of magnitude for our experiment are $\lambda_{0} \sim 0.4 \mu \mathrm{m}, d \sim 100 \mu \mathrm{m}, \Omega_{\mathrm{c}} \sim 1 \mathrm{sr}$ and thus $\sigma_{c} / S \sim 1.5 \times 10^{-5}$. The resulting signal must be compared to the fluctuations of the background $C_{12}$, due to uncorrelated detections (Eq. (30)). Assuming that the counting rate of the photodetector is $N$ (in s ${ }^{-1}$ ), the fluctuations for a counting time $T$ will be :

$$
B \sim \sqrt{N^{2} \tau_{\mathrm{c}} T}
$$

while the signal is :

$$
S \sim \frac{\sigma_{\mathrm{c}}}{S} N^{2} \tau_{\mathrm{c}} T
$$

The signal-to-noise-ratio is thus :

$$
\frac{S}{B} \sim N \tau_{\mathrm{c}} \frac{\sigma_{\mathrm{c}}}{S} \sqrt{\frac{T}{\tau_{\mathrm{c}}}} .
$$

In our case, $N \sim 5000 \mathrm{~s}^{-1}, \tau_{\mathrm{c}} \sim 10^{-9} \mathrm{~s}$, and $\sigma_{\mathrm{c}} / S \sim 10^{-5}$; the counting time $T$ corresponding to $S / B \sim 1$ would be 10000 years! On the other hand, the same values except $\sigma_{\mathrm{c}} / S \sim 1$ yield $T \sim 1 \mathrm{~min}$. Similar equations hold for spectrum-analysing experiments [19].

In order to increase $\sigma_{\mathrm{c}} / S$, it is not sufficient to reduce $\Omega_{\mathrm{c}}$, since the number of detected photons $N$ would decrease, with no gain on the signal-to-noise ratio. Actually, one must increase the ratio $N / d^{2} \Omega_{\mathrm{c}}$, i.e. the intensity and luminance of the source, without changing the coherence time $\tau_{\mathrm{c}}$. In conclusion, this kind of C.W. experiment appears 
hardly feasible, and would require an experimental configuration completely different from the one described in this paper.

\section{Conclusion.}

Since the completion of our experimental work [1], theoretical analysis of the time-resolved fluorescence induced by molecular photodissociation have been proposed by Kurizki and Ben-Reuven [21] and by Diebold [22], using formalisms different from the one proposed in this paper, but giving essentially the same results. However, let us emphasize that our Heisenberg equations approach gives some clear physical insights into the interference effect. In particular, the interference term between the contributions of the two dipoles appears very clearly ; in our experimental conditions, this term yields the main effect, and the modification of the radiative damping is not directly observed. Moreover, crossed effects involving several molecules were easily taken into account.

Further developments in our experiment would involve the use of a tunable laser, in order to analyse more precisely the molecular bound or continuum states; such an analysis is beyond the scope of the present paper.

As a conclusion, we shall comment briefly about the symmetrization of the state for two calcium atoms ; indeed, the initial state of the pair of atoms may be considered as a «delocalized » quantum state [23], with some analogy with non-factorizable states leading to Einstein-Podolsky-Rosen correlations. However, let us emphasize that in our case the complete symmetrization of the wavefunctions would not change any physical prediction: the interference effect is due to the original molecular symmetry, and not to the boson or fermion nature of the atoms. Moreover, the observed effect would be the same if we could perform the experiment by photodissociating a molecule formed of two nonidentical atoms [24].

The authors acknowledge fruitful discussions with A. Aspect.

\section{Appendix I.}

EQUATIONS FOR THE EVOLUTION OF THE ATOMIC OPERATORS. - In this appendix we derive equation (16) from equation (15). Using standard methods for the calculation of spontaneous emission [3], we can write :

$$
\begin{aligned}
\frac{\mathrm{d}}{\mathrm{d} t} \tilde{S}_{a}^{-}(t)\left|\psi_{\mathrm{in}}\right\rangle= & \sum_{\lambda} g_{\lambda}^{2}\left(2 \tilde{S}_{\mathrm{a}}^{+} \tilde{S}_{\mathrm{a}}^{-}-1\right) \times \\
& \times\left(\tilde{S}_{\mathrm{a}}^{-}+\tilde{S}_{\mathrm{b}}^{-} \mathrm{e}^{i \mathbf{k}_{\lambda}\left(\mathbf{R}_{\mathrm{a}}-\mathbf{R}_{\mathrm{b}}\right)}\right) \\
& \times \int_{0}^{t} \mathrm{e}^{i\left(\omega_{\lambda}-\omega_{0}\right)\left(t^{\prime}-t\right)} \mathrm{d} t^{\prime}\left|\psi_{\text {in }}\right\rangle
\end{aligned}
$$

$$
\begin{aligned}
\frac{\mathrm{d}}{\mathrm{d} t} \tilde{S}_{\mathrm{b}}^{-}(t)\left|\psi_{\text {in }}\right\rangle=\sum_{\lambda} g_{\lambda}^{2} & \left(2 \tilde{S}_{\mathrm{b}}^{+} \tilde{S}_{\mathrm{b}}^{-}-1\right) \times \\
& \times\left(\tilde{S}_{\mathrm{a}}^{-} \mathrm{e}^{i \mathbf{k}_{\lambda}\left(\mathbf{R}_{\mathrm{b}}-\mathbf{R}_{\mathrm{a}}\right)}+\tilde{S}_{\mathrm{b}}^{-}\right) \\
& \times \int_{0}^{t} \mathrm{e}^{i\left(\omega_{\lambda}-\omega_{0}\right)\left(t^{\prime}-t\right)} \mathrm{d} t^{\prime}\left|\psi_{\text {in }}\right\rangle .
\end{aligned}
$$

We then use the relation:

$$
\begin{aligned}
\lim _{t \rightarrow \infty} \int_{0}^{t} \mathrm{e}^{i\left(\omega_{\lambda}-\omega_{0}\right)\left(t^{\prime}-t\right)} \mathrm{d} t^{\prime} & \\
& =\pi \delta\left(\omega_{\lambda}-\omega_{0}\right)-i P \frac{1}{\omega_{\lambda}-\omega_{0}} \\
& =\lim _{\varepsilon \rightarrow 0} \frac{-i}{\omega_{\lambda}-\omega_{0}-i \varepsilon}
\end{aligned}
$$

Two integral over $\lambda$ must be calculated :

$$
\begin{aligned}
J_{1} & =\sum_{\lambda} g^{2}\left(\pi \delta\left(\omega_{\lambda}-\omega_{0}\right)-i P\left(\frac{1}{\omega_{\lambda}-\omega_{0}}\right)\right) \\
& =\frac{\Gamma}{2}+i \Delta
\end{aligned}
$$

$\Gamma$ is the inverse of the atomic lifetime already given, and $\Delta$ is the level shift, which cannot be calculated correctly for a two-level system, and will be omitted in the following (indeed, the total field radiated by a dipole at its own emplacement is not described correctly by our model). The second relevant integral is :

$$
\begin{aligned}
J_{2}= & \sum_{\lambda} g_{\lambda}^{2} \mathrm{e}^{i \mathbf{k}_{\lambda} \cdot\left(\mathbf{R}_{\mathrm{a}}-\mathbf{R}_{\mathrm{b}}\right)} \times \\
& \times\left(\pi \delta\left(\omega_{\lambda}-\omega_{0}\right)-i P \frac{1}{\omega_{\lambda}-\omega_{0}}\right) \\
= & \lim _{\varepsilon \rightarrow 0} \sum_{\lambda} g_{\lambda}^{2} \frac{-i \mathrm{e}^{i \mathbf{k}_{\lambda} \cdot \delta}}{\left(\omega_{\lambda}-\omega_{0}\right)-i \varepsilon}
\end{aligned}
$$

where $\boldsymbol{\delta}=\mathbf{R}_{\mathrm{a}}-\mathbf{R}_{\mathrm{b}}$.

The angular integrals may be calculated conveniently in the molecular frame, where the axis $\mathrm{OZ}$ is parallel to $\delta$. Two cases are considered : $\Sigma$-excited state, where the dipole $d_{e g}$ is also parallel to $\mathrm{OZ}$, and $\pi$-excited state, where the dipole $d_{\mathrm{eg}}$ is parallel to some axis $\mathrm{O} X$. We obtain :

$$
J_{2}=\lim _{\varepsilon^{\prime} \rightarrow 0} \frac{i \Gamma}{2 \pi k_{0}^{3}} \int \mathrm{d} k k^{3} f(k \delta)\left(\frac{1}{k-k_{0}-i \varepsilon^{\prime}}\right)
$$

where

$$
\begin{gathered}
f^{\pi}(u)=\frac{3}{2}\left(\frac{\sin u}{u}-\frac{\sin u}{u^{3}}+\frac{\cos u}{u^{2}}\right) \\
f^{\Sigma}(u)=3\left(-\frac{\cos u}{u^{2}}+\frac{\sin u}{u^{3}}\right) .
\end{gathered}
$$

The above integral can easily be calculated using contour integration, and we obtain : 


$$
J_{2}=\frac{\Gamma}{2}\left\{f\left(k_{0} \delta\right)+i g\left(k_{0} \delta\right)\right\}
$$

where

$$
\begin{aligned}
& g^{\pi}(u)=\frac{3}{2}\left(-\frac{\cos u}{u}+\frac{\sin u}{u^{2}}+\frac{\cos u}{u^{3}}\right) \\
& g^{\Sigma}(u)=-3\left(\frac{\sin u}{u^{2}}+\frac{\cos u}{u^{3}}\right) .
\end{aligned}
$$

Note that

$$
\lim _{u \rightarrow 0} f^{\pi}(u)=\lim _{u \rightarrow 0} f^{\Sigma}(u)=1
$$

while :

$$
\lim _{u \rightarrow 0} g^{\pi}(u)=-\frac{1}{2} \lim _{u \rightarrow 0} g^{\Sigma}(u) \sim \frac{3}{2} \frac{1}{u^{3}} .
$$

Using equations (I.2) and (I.3) in (I.1), we obtain easily equation (16), which represent coupled equations for the evolution of $\tilde{S}_{\mathrm{a}}^{-}(t)$ and $\tilde{S}_{\mathrm{b}}^{-}(t)$.

\section{Appendix II.}

TIME EVOLUTION OF THE ATOMIC OPERATORS. The general solution of equation (16) may be obtained in the four-dimensional space with vector basis $\left(\left|e_{a} e_{b}\right\rangle,\left|e_{a}, g_{b}\right\rangle,\left|g_{a}, e_{b}\right\rangle,\left|g_{a} g_{b}\right\rangle\right)$. It is more convenient to calculate the time evolution of the operators :

$$
\begin{aligned}
& \tilde{\boldsymbol{\Sigma}}^{+}=\tilde{S}_{\mathrm{a}}^{+}+\tilde{S}_{\mathrm{b}}^{+} \\
& \tilde{\boldsymbol{\Delta}}^{+}=\tilde{S}_{\mathrm{a}}^{+}-\tilde{S}_{\mathrm{b}}^{+} .
\end{aligned}
$$

From equation (16), we obtain :

$$
\begin{aligned}
& \frac{\mathrm{d}}{\mathrm{d} t}\left(\tilde{\Sigma}^{+} \tilde{\Sigma}^{-}\right)=-\Gamma\left(1+f\left(k_{0} \delta\right)\right) \tilde{\Sigma}^{+} \tilde{\Sigma}^{-}+ \\
&+\Gamma f\left(k_{0} \delta\right) \tilde{\Sigma}^{+} \tilde{\Sigma}^{+} \tilde{\Sigma}^{-} \tilde{\Sigma}^{-} \\
& \frac{\mathrm{d}}{\mathrm{d} t}\left(\tilde{\Delta}^{+} \tilde{\Delta}^{-}\right)=-\Gamma\left(1-f\left(k_{0} \delta\right)\right) \tilde{\Delta}^{+} \tilde{\Delta}^{-}- \\
&-\Gamma f\left(k_{0} \delta\right) \tilde{\Sigma}^{+} \tilde{\Sigma}^{+} \tilde{\Sigma}^{-} \tilde{\Sigma}^{-} \\
& \frac{\mathrm{d}}{\mathrm{d} t}\left(\tilde{\Sigma}^{+} \tilde{\Delta}^{-}\right)=-\Gamma\left(1-i g\left(k_{0} \delta\right)\right) \tilde{\Sigma}^{+} \tilde{\Delta}^{-} \\
& \frac{\mathrm{d}}{\mathrm{d} t}\left(\tilde{\Delta}^{+} \tilde{\Sigma}^{-}\right)=-\Gamma\left(1+i g\left(k_{0} \delta\right)\right) \tilde{\Delta}^{+} \tilde{\Sigma}^{-} \\
& \frac{\mathrm{d}}{\mathrm{d} t}\left(\tilde{\Sigma}^{+} \tilde{\Sigma}^{+} \tilde{\Sigma}^{-} \tilde{\Sigma}^{-}\right)=-2 \Gamma \tilde{\Sigma}^{+} \tilde{\Sigma}^{+} \tilde{\Sigma}^{-} \tilde{\Sigma}^{-}
\end{aligned}
$$

We have cancelled products or combinations of operators which are equal to zero inside the considered space.

Equation (11.4) yields immediately :

$$
\left(\tilde{\Sigma}^{+} \tilde{\Sigma}^{+} \tilde{\Sigma}^{-} \tilde{\Sigma}^{-}\right)(t)=\sigma_{0} \mathrm{e}^{-2 \Gamma t}
$$

where $\sigma_{0}$ is a constant depending on the initial state.
We shall restrict ourselves in the following to a single excitation of the system, i.e. $\sigma_{0}=0$.

The case of doubly excited systems has been considered in great detail by Richter [25], when the internuclear distance $\delta$ is fixed. Let us notice that [25] uses a master equation approach and the quantum regression theorem. Obviously, the same results may be obtained using our Heisenberg equations approach.

Equations (11.2) and (11.3) then yield :

$$
\begin{aligned}
\left(\tilde{\Sigma}^{+} \tilde{\Sigma}^{-}\right)(t)= & \left(\tilde{\Sigma}^{+} \tilde{\Sigma}^{-}\right)_{0} \times \\
& \times \exp \left(-\Gamma \int_{0}^{t}\left(1+f\left(k_{0} \delta\right)\right) \mathrm{d} t^{\prime}\right)
\end{aligned}
$$

$$
\begin{aligned}
\left(\tilde{\Delta}^{+} \tilde{\Delta}^{-}\right)(t)= & \left(\tilde{\Delta}^{+} \tilde{\Delta}^{-}\right)_{0} \times \\
& \times \exp \left(-\Gamma \int_{0}^{t}\left(1-f\left(k_{0} \delta\right)\right) \mathrm{d} t^{\prime}\right)
\end{aligned}
$$

$\left(\tilde{\Sigma}^{+} \tilde{\Delta}^{-}\right)(t)=\left(\tilde{\Sigma}^{+} \tilde{\Delta}^{-}\right)_{0} \times$

$$
\times \exp \left(-\Gamma \int_{0}^{t}\left(1-i g\left(k_{0} \delta\right)\right) \mathrm{d} t^{\prime}\right)
$$

$$
\begin{aligned}
\left(\tilde{\Delta}^{+} \tilde{\Sigma}^{-}\right)(t)= & \left(\tilde{\Delta}^{+} \tilde{\Sigma}^{-}\right)_{0} \times \\
& \times \exp \left(-\Gamma \int_{0}^{t}\left(1+i g\left(k_{0} \delta\right)\right) \mathrm{d} t^{\prime}\right)
\end{aligned}
$$

From these equations, any normal ordered combination of the operators can be easily obtained.

- For the initial state $\frac{1}{\sqrt{2}}\left(\left|\mathrm{~g}_{\mathrm{a}} \mathrm{e}_{\mathrm{b}}\right\rangle+\varepsilon\left|\mathrm{e}_{\mathrm{a}} \mathrm{g}_{\mathrm{b}}\right\rangle\right)$, we get easily $\left\langle\tilde{\Sigma}^{+} \tilde{\Sigma}^{-}\right\rangle_{0}=(1+\varepsilon),\left\langle\tilde{\Delta}^{+} \tilde{\Delta}^{-}\right\rangle_{0}=$ $(1-\varepsilon)$, while $\left\langle\tilde{\Sigma}^{+} \tilde{\Delta}^{-}\right\rangle_{0}$ and $\left\langle\tilde{\Delta}^{+} \tilde{\Sigma}^{-}\right\rangle_{0}$ are equal to zero; these results may be recasted in the following form used in equation (17) :

$$
\begin{aligned}
\left\langle S_{\mathrm{a}}^{+} S_{\mathrm{a}}^{-}\right\rangle=\left\langle S_{\mathrm{b}}^{+}\right. & \left.S_{\mathrm{b}}^{-}\right\rangle=\frac{1}{2} \times \\
& \times \exp \left(-\Gamma \int_{0}^{t}\left(1+\varepsilon f\left(k_{0} \delta\right)\right) \mathrm{d} t^{\prime}\right)
\end{aligned}
$$

$$
\begin{aligned}
\left\langle S_{\mathrm{a}}^{+} S_{\mathrm{b}}^{-}\right\rangle=\left\langle S_{\mathrm{b}}^{+}\right. & \left.S_{\mathrm{a}}^{-}\right\rangle=\frac{\varepsilon}{2} \times \\
& \times \exp \left\langle-\Gamma \int_{0}^{t}\left(1+\varepsilon f\left(k_{0} \delta\right)\right) \mathrm{d} t^{\prime}\right\rangle .
\end{aligned}
$$

\section{Appendix III.}

EQUATIONS FOR THE EVOLUTION OF THE IMPULSION OPERATORS. - System (18) can be rewritten using the approximations of Appendix I. Two new integrals appear : 


$$
\begin{gathered}
J_{1}^{\prime}=\sum_{\lambda} g_{\lambda}^{2} \hbar \int_{0}^{t} \mathrm{e}^{i\left(\omega_{\lambda}-\omega_{0}\right)\left(t^{\prime}-t\right)} \mathrm{d} t^{\prime} \\
J_{2}^{\prime}=\sum_{\lambda} g_{\lambda}^{2} \hbar \mathbf{k}_{\lambda} \mathrm{e}^{i \mathbf{k}_{\lambda}\left(\mathbf{R}_{\mathrm{a}}-\mathbf{R}_{\mathrm{b}}\right)} \int_{0}^{t} \mathrm{e}^{i\left(\omega_{\lambda}-\omega_{0}\right)\left(t^{\prime}-t\right)} \mathrm{d} t^{\prime}
\end{gathered}
$$

The angular integration over $\mathbf{k}$ yields $J_{1}^{\prime}=0$. We define $\operatorname{grad}_{\boldsymbol{\delta}}$ where $\boldsymbol{\delta}=\mathbf{R}_{\mathrm{a}}-\mathbf{R}_{\mathrm{b}}$ as the gradient relative to the composants of $\left(\mathbf{R}_{\mathrm{a}}-\mathbf{R}_{\mathrm{b}}\right) ; J_{2}^{\prime}$ can be transformed :

$$
\begin{aligned}
J_{2}^{\prime} & =\frac{\hbar}{i} \operatorname{grad}_{\delta} J_{2} \\
& =\frac{\hbar \Gamma}{2 i} \operatorname{grad}_{\delta}\left(f\left(k_{0} \delta\right)+i g\left(k_{0} \delta\right)\right)
\end{aligned}
$$

where $J_{2}, f\left(k_{0} \delta\right)$ and $g\left(k_{0} \delta\right)$ have been defined in Appendix I.

Recombining the two equations of system (18), we obtain finally:

$$
\begin{aligned}
\frac{\mathrm{d}}{\mathrm{d} t}\left(\mathbf{P}_{\mathrm{a}}+\mathbf{P}_{\mathrm{b}}\right)=\left(\tilde{S}_{\mathrm{a}}^{+} \tilde{S}_{\mathrm{b}}^{-}-\tilde{S}_{\mathrm{b}}^{+}\right. & \left.\tilde{S}_{\mathrm{a}}^{-}\right) \times \\
& \times i \hbar \Gamma \operatorname{grad}_{\delta}\left(f\left(k_{0} \delta\right)\right) \\
\frac{\mathrm{d}}{\mathrm{d} t}\left(\mathbf{P}_{\mathrm{a}}-\mathbf{P}_{\mathrm{b}}\right)=\left(\tilde{S}_{\mathrm{a}}^{+} \tilde{S}_{\mathrm{b}}^{-}+\tilde{S}_{\mathrm{b}}^{+}\right. & \left.\tilde{S}_{\mathrm{a}}^{-}\right) \times \\
& \times \hbar \Gamma \operatorname{grad}_{\delta}\left(g\left(k_{0} \delta\right)\right) .
\end{aligned}
$$

The first equation means that the average recoil is not zero if the excitation is anisotropic (e.g. one excited atom, one ground state atom). The second equation gives the interaction between the two atoms, which is discussed in $\S 1.4$ for our initial state $\left|\psi_{\text {in }}\right\rangle$.

\section{Appendix IV.}

ANGUlar AVERAging. - The recoil direction of the atoms is characterized by the angle $\theta, \phi$ defined in figure 1. The excited dipole is obtained as the projection of the dissociating electric field $\mathbf{E}_{\mathrm{L}}$ onto the molecular axis for a $\Sigma$-state, and onto a plane orthogonal to the molecular axis for a $\Pi$-state. Taking $\mathrm{E}_{\mathrm{L}}$ along $\mathrm{O} x$, the corresponding weighting functions are :

$$
\begin{aligned}
& W_{\mathrm{e}}^{\Sigma}(\theta, \phi)=\sin ^{2} \theta \cos ^{2} \phi \\
& W_{\mathrm{e}}^{I}(\theta, \phi)=\sin ^{2} \theta \sin ^{2} \phi+\cos ^{2} \theta .
\end{aligned}
$$

The detected intensity is obtained as the projection of the excited dipole onto $O x$ (// polarization) or $\mathrm{Oy}$ $\left(\perp\right.$ polarization). Denoting $W_{\mathrm{d}}(\theta, \phi)$ the corresponding weighting function, the angular average of the interference term $\left(1+\varepsilon \cos \left(\Omega_{0} \tau \cos \theta\right)\right)$ is given by :

$$
\begin{aligned}
I(\alpha)=\frac{1}{4 \Pi} \iint & \mathrm{d} \phi \mathrm{d} \theta \sin \theta W_{\mathrm{e}}(\theta, \phi) \times \\
& \times W_{\mathrm{d}}(\theta, \phi)(1+\varepsilon \cos (\alpha \cos \theta))
\end{aligned}
$$

where $\alpha=\Omega_{0} \tau=\omega_{0} v \tau / c$.

We give below the expressions of $I(\alpha)$ for the states which are coupled to the $\mathrm{X}^{1} \Sigma_{\mathrm{g}}^{+}$ground state by a dipolar transition, i.e. the ${ }^{1} \Sigma_{u}$ and ${ }^{1} \Pi_{u}$ excited states $(\varepsilon=+1)$.

$$
\begin{aligned}
I_{\Gamma} \Gamma^{\Sigma}(\alpha)=3 I_{\perp}^{\Sigma}(\alpha)=3\left(\frac{1}{15}+\right. & \left(-\frac{1}{\alpha^{3}}+\frac{3}{\alpha^{5}}\right) \times \\
& \left.\times \sin \alpha-\frac{3}{\alpha^{4}} \cos \alpha\right)
\end{aligned}
$$

$I^{\Pi}(\alpha)=\frac{8}{15}+\left(\frac{1}{\alpha}-\frac{5}{\alpha^{3}}+\frac{9}{\alpha^{5}}\right) \times$

$$
\times \sin \alpha+\left(\frac{2}{\alpha^{2}}-\frac{9}{\alpha^{4}}\right) \cos \alpha
$$

$I_{\perp}^{\Pi}(\alpha)=\frac{1}{15}+\left(-\frac{1}{\alpha^{3}}+\frac{3}{\alpha^{5}}\right) \sin \alpha-\frac{3}{\alpha^{4}} \cos \alpha$.

Note that the corresponding polarization $\left(I_{\|}-I_{\perp}\right) /\left(I_{\|}+I_{\perp}\right)$ is equal to $1 / 2$ for a $\Sigma$-state, and oscillates slightly around the value $7 / 9$ for a $\Pi$-state.

The values given in the text are obtained from a similar averaging, but taking $W_{\mathrm{e}}(\theta, \phi)=1$ (isotropic excited molecular state distribution).

\section{References}

[1] Grangier, P., Aspect, A. and Vigué, J., Phys. Rev. Lett. 54 (1985) 418.

[2] This expression of the coupling is rigorously demonstrated in : C. CoHEN-TANNOUDJI, Ecole d'Eté des Houches, 1982, eds. G. Grynberg and R. Stora (North Holland) 1984. It is shown in this reference that the interaction term involves the electric induction rather than the electric field. However, the mathematical operator associated with this electric induction is identical to the one defined by equation (5) outside the dipoles own emplacements.
[3] Moreover, we must restrict ourselves to the calculation of normal-ordered products of operators. For a discussion, see reference [2], and : R. R. PURI, J. Opt. Soc. Am. B 2 (1985) 447.

[4] Loudon, R., The Quantum Theory of Light, $2^{\text {nd }}$ Edition (Clarendon, Oxford), 1983. See also reference [3].

[5] Glauber, R. J., in Quantum Optics and Electronics, eds. C. de Witt, A. Blandin and C. CohenTannoudji (Gordon and Breach, 1965).

[6] Vigué, J., Beswick, J. A. and Broyer, M., J. Physique 44 (1983) 1225. 
[7] Diebold, G. J., Phys. Rev. Lett. 51 (1983) 1344.

[8] This is true for an optically thin source (no reabsorption of the emitted field). Some consequences of reabsorption are discussed in Part III.

[9] Rosenkrantz, M. E., Krauss, M., Stevens, J., Chem. Phys. Lett. 89 (1982) 4.

[10] Hofmann, R. T., Harris, D. O., J. Chem. Phys. 81 (1984) 1047.

[11] Bondybey, V. E., English, J. H., Chem. Phys. Lett. 111 (1984) 195.

[12] Vidal, C. R., J. Chem. Phys. 72 (1980) 1864.

[13] Miller, J. C., Mowery, R. L., Krausz, E. R., JacoBs, S. M., KIM, H. W., SchatZ, P. N., Andrews, L., J. Chem. Phys. 74 (1981) 6349.

[14] Vigué, J., Grangier, P., Roger, G. and Aspect, A., J. Physique Lett. 42 (1981) L-531.

[15] At long distance the potential curve of this ${ }^{1} \Pi_{\mathrm{u}}$ state is dominated by the (repulsive) long range resonance dipole interaction (see section 1.4 of the present paper or G. W. KING, J. H. VAN VleCK, Phys. Rev. 55 (1939) 1165). But at shorter internuclear distance the ${ }^{1} \Pi_{u}$ state may interact with a state of the same symmetry and of ionic nature, i.e. dissociating in $\mathrm{Ca}^{+}\left({ }^{2} \mathrm{~S}\right)+$ $\mathrm{Ca}^{-}\left({ }^{2} \mathrm{P}\right)$. The same phenomenon has been predicted in $\mathbf{M g}_{2}$ (W. J. STEVEnS, M. KRAuss, $J$. Chem. Phys. 67 (1977) 1977) and observed in $\mathrm{Be}_{2}$ (V. E. Bondybey, Chem. Phys. Letters 109 (1984) 436). However, a rough estimation with zero electronic affinity for $\mathrm{Ca}^{-}$yields an upper limit for the crossing point at an internuclear distance about $5 \AA$, and the ${ }^{1} \Pi_{u}$ state is probably weakly affected in the range of interest ( $R$. Grice, D. R. Herschbach, Mol. Phys. 27 (1974) 159).

[16] Child, M. S., Can. J. Phys. 53 (1975) 1830.

[17] MeIwes-Broer, K. H., Thesis (Bielefeld University 1983) unpublished.

[18] Aspect, A. and Grangier, P., Lett. Nuovo Cim. 43 (1985) 345.

[19] Forrester, A. T., Gudmundsen, R. A. and JohnSON, P. O., Phys. Rev. 99 (1955) 1961.

[20] Nienhuis, G., Phys. Rev. A 31 (1985) 1929.

[21] Kurizki, G. and Ben-Reuven, A., Phys. Rev. A 32 (1985) 2560.

[22] Diebold, G. J., Phys. Rev. A 32 (1985) 1458.

[23] Horne, A. A., Zeilinger, A. and Bernstein, H. J., Private Communication.

[24] The two atoms shoud have the same dipole moment, and resonance frequencies differing by much less than the modulation frequency

$$
\Omega_{0} / 2 \pi=v / \lambda_{0} ;
$$

a possible system would be the ${ }^{40} \mathrm{Ca}-{ }^{42} \mathrm{Ca}$ molecule, with an isotopic shift on the resonance line equal to $390 \mathrm{MHz}$ (A. ANDl et al., Phys. Rev. C 26 (1982) 2194), while in our experiment $\Omega_{0} / 2 \pi=2500 \mathrm{MHz}$. See also reference [21].

[25] Richter, Th., Ann. Physik Leipz. 36 (1979) 266.

[26] Cohen-Tannoudj, C., Diu, B. et Lalö, F., Mécanique Quantique (Hermann, Paris) 1977, p. 1128 and reference therein.

[27] Dicke, R. H., Phys. Rev. 93 (1954) 99. 